\title{
Dendritic cells expressing immunoreceptor CD300f are critical for controlling chronic gut inflammation
}

\author{
Ha-Na Lee, ${ }^{1}$ Linjie Tian, ${ }^{1}$ Nicolas Bouladoux, ${ }^{2,3}$ Jacquice Davis, ${ }^{2}$ Mariam Quinones, ${ }^{4}$ Yasmine Belkaid, ${ }^{2,3}$ John E. Coligan, ${ }^{1}$ \\ and Konrad Krzewski' \\ ${ }^{1}$ Receptor Cell Biology Section, Laboratory of Immunogenetics, National Institute of Allergy and Infectious Diseases, NIH, Rockville, Maryland, USA. ${ }^{2}$ NIAID Microbiome Program, ${ }^{3}$ Mucosal Immunology \\ Section, and ${ }^{4}$ Bioinformatics and Computational Bioscience Branch, National Institute of Allergy and Infectious Diseases, NIH, Bethesda, Maryland, USA.
}

\begin{abstract}
Proinflammatory cytokine overproduction and excessive cell death, coupled with impaired clearance of apoptotic cells, have been implicated as causes of failure to resolve gut inflammation in inflammatory bowel diseases. Here we have found that dendritic cells expressing the apoptotic cell-recognizing receptor CD300f play a crucial role in regulating gut inflammatory responses in a murine model of colonic inflammation. CD300f-deficient mice failed to resolve dextran sulfate sodium-induced colonic inflammation as a result of defects in dendritic cell function that were associated with abnormal accumulation of apoptotic cells in the gut. CD300f-deficient dendritic cells displayed hyperactive phagocytosis of apoptotic cells, which stimulated excessive TNF- $\alpha$ secretion predominantly from dendritic cells. This, in turn, induced secondary IFN- $\gamma$ overproduction by colonic $\mathrm{T}$ cells, leading to prolonged gut inflammation. Our data highlight a previously unappreciated role for dendritic cells in controlling gut homeostasis and show that CD300f-dependent regulation of apoptotic cell uptake is essential for suppressing overactive dendritic cell-mediated inflammatory responses, thereby controlling the development of chronic gut inflammation.
\end{abstract}

\section{Introduction}

Inflammatory bowel diseases (IBDs), including ulcerative colitis and Crohn's disease, are chronic relapsing inflammatory disorders within the intestinal lumen arising from a combination of immunological, environmental, and genetic factors $(1,2)$. Although the etiology of IBDs remains unclear, a sustained overproduction of proinflammatory cytokines and excessive cell death coupled with the impaired clearance of apoptotic cells (ACs) in the intestinal lamina propria have been implicated as the primary reasons for failure to resolve acute inflammation in the gut (3). High levels of apoptosis have been observed in the intestinal epithelium of ulcerative colitis patients (4-6). Moreover, several animal studies confirm the role of apoptosis in IBD pathogenesis. For instance, deficiencies in XBP1, NF- $\kappa \mathrm{B}$, or FADD have been shown to lead to apoptosis of colonic epithelial cells and development of intestinal inflammation (7-10). Therefore, dysregulated function of innate immune cells that are responsible for phagocytosis of ACs (efferocytosis), such as macrophages and dendritic cells (DCs), could augment IBD pathogenesis. Intriguingly, IBD patients display higher frequencies of DCs positive for several activation and maturation markers in the lamina propria of inflamed colon tissues, and IBD DCs produce significantly more proinflammatory cytokines than DCs from healthy controls (11-13). Although these studies indi-

Authorship note: J.E. Coligan and K. Krzewski are co-senior authors and contributed equally to this work.

Conflict of interest: The authors have declared that no conflict of interest exists.

Submitted: July 13, 2016; Accepted: February 16, 2017.

Reference information: J Clin Invest. 2017;127(5):1905-1917.

https://doi.org/10.1172/JCI89531 cate that DCs have a function in IBD pathogenesis, their role in the inflammatory cascade leading to intestinal inflammation, and its association with AC clearance, has not been fully elucidated.

The best-known "eat me" signal displayed on the AC surface, phosphatidylserine (PS), is utilized by phagocytes to engulf ACs (14, 15). CD300f (also known as CLM-1), expressed mainly by myeloid cells, is a PS-recognizing receptor with a single IgV-like extracellular domain and an intracellular tail containing both tyrosine-based activating and inhibitory motifs, which regulates efferocytosis by professional phagocytes (16-18). Recently we have demonstrated that, in contrast to CD300f function on macrophages, its expression inhibits efferocytosis by DCs (18). However, how CD300f-mediated efferocytosis, especially by DCs, regulates acute and/or chronic inflammatory responses in vivo has not been determined.

In this report, we demonstrate that $C D 300 f^{\prime-}$ mice, in marked contrast to WT mice, fail to resolve colonic inflammation. CD30Of/mice show significant accumulation of ACs in the inflamed colon, likely due to impaired macrophage efferocytosis, making ACs more available to CD300f-deficient DCs that have significantly enhanced efferocytic capability. Most importantly, engulfment of ACs by CD300f-deficient DCs stimulates them to produce TNF- $\alpha$, which in turn induces overproduction of IFN- $\gamma$, mainly by $\mathrm{T}$ cells, leading to a failure to resolve colonic inflammation in a timely manner. Thus, we demonstrate a critical role for CD300f expression by DCs in regulating chronic inflammation associated with IBDs.

\section{Results}

CD300f deficiency accelerates colitis and suppresses resolution of colonic inflammation. To investigate to what extent CD300f functions in regulation of colonic inflammation, $C D 300 \mathrm{f}^{\mathrm{t}+}$ and 
A
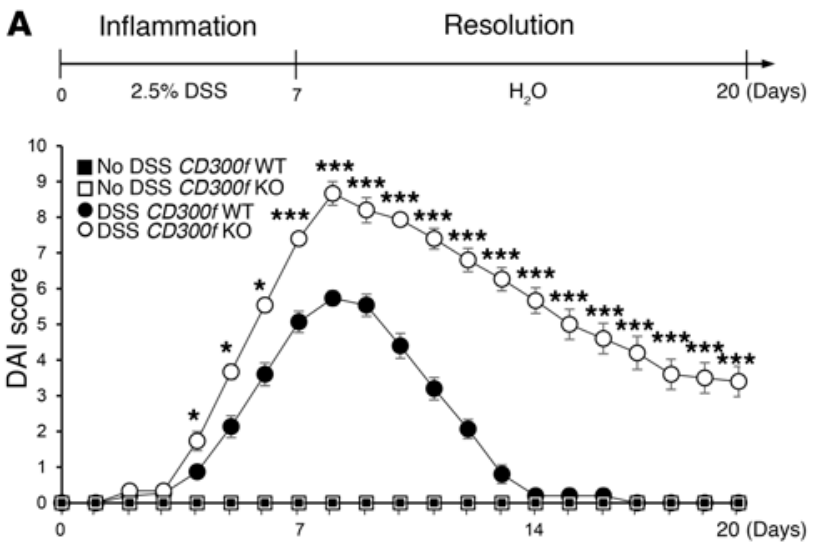

B
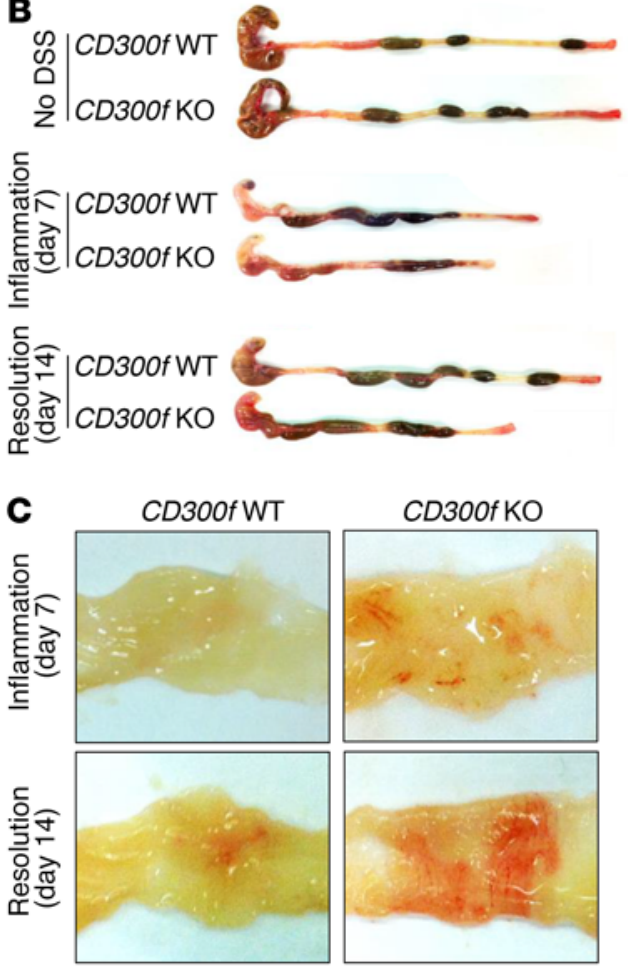

D
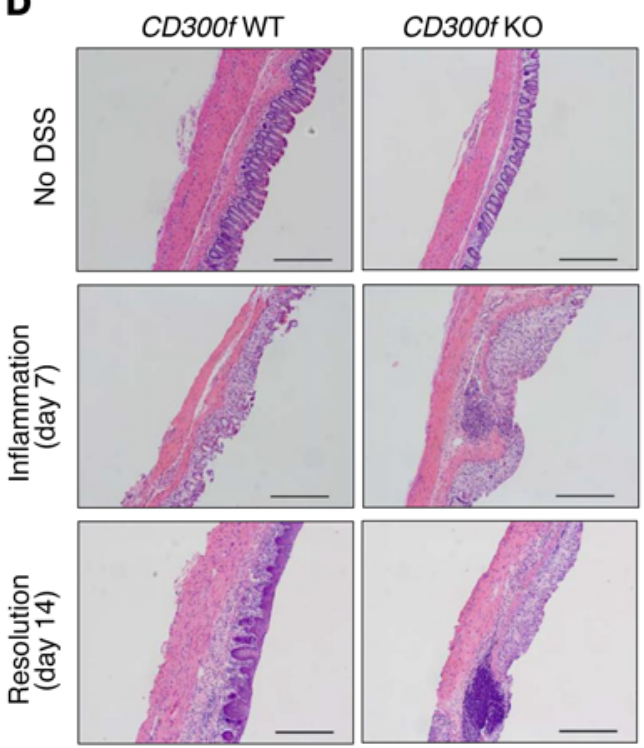
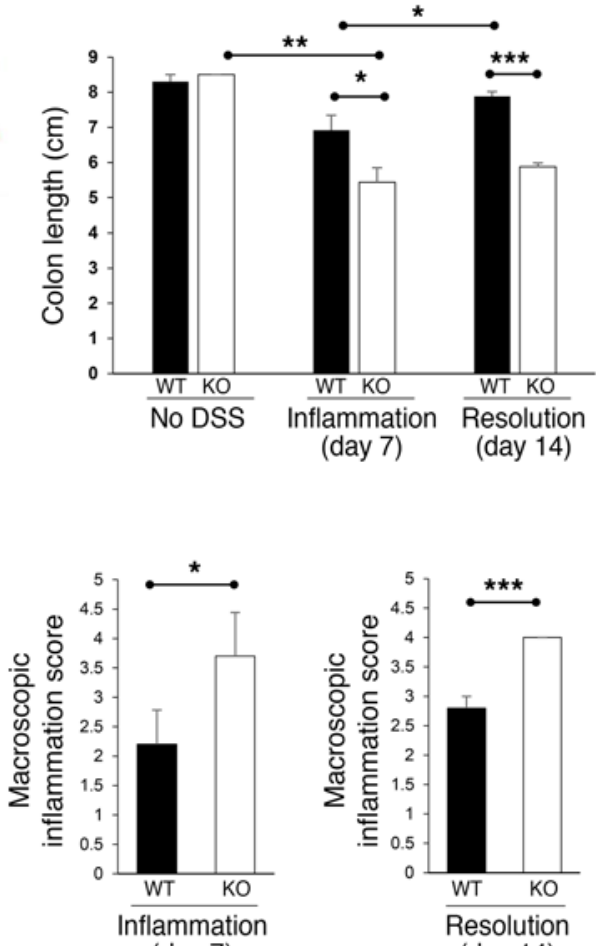

(day 7 )

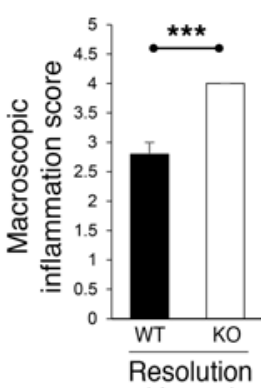

(day 14)

Figure 1. CD300f deficiency leads to failure in resolution of colonic inflammation. (A-D) CD300f $\mathrm{f}^{\prime+}$ (CD300f WT) or CD300f $\mathrm{f}^{-1-}$ (CD300f KO) mice were given drinking water containing $2.5 \%$ DSS for 7 days, followed by unadulterated water for another 13 days (20 days total). "No DSS" indicates mice without DSS administration. DAI was scored during DSS administration, as described in Methods (A). On day 7 (Inflammation) and day 14 (Resolution), the colon length (B), macroscopic inflammation score (C), and microscopic inflammation score (D) were determined. The pictures in $\mathbf{B}-\mathbf{D}$ are representative images from the indicated mice; note that $C D 300 f^{-1-}$ mice have shortened colons with loose stools (B), evident hyperemia (C), and destruction of colonic architecture with elevated cell infiltration (D). Scale bars in H\&E staining images: 500 $\mu \mathrm{m}$. Graphs show data quantification with means; error bars represent SEM $(n=3$ in control, $n=15$ in day $7, n=15$ in day 14). Twotailed paired Student's $t$ test was used to determine statistical significance $\left({ }^{*} P<0.05,{ }^{* *} P<0.01,{ }^{* * *} P<0.001\right)$.
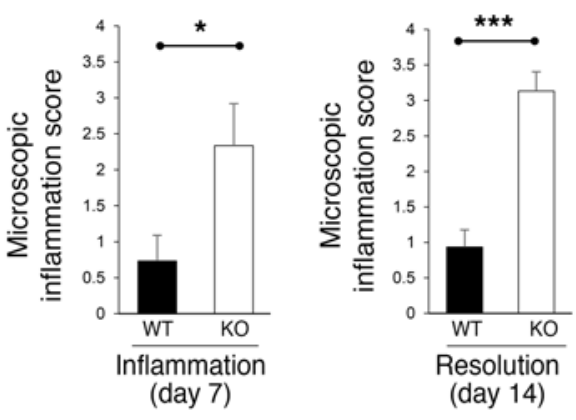
CD300f/- mice were given drinking water containing $2.5 \%$ dextran sulfate sodium (DSS) for 7 days, which causes severe damage to the colonic epithelium that triggers colonic inflammation (19), followed by normal drinking water for an additional 13 days to allow DSS-induced intestinal inflammation to subside. The disease activity index (DAI) score reached maximal value in both $\mathrm{CD} 00 \mathrm{f}^{+/+}$and $C D 300 f^{-/-}$mice on day 8, with $C D 300 f^{-/-}$ mice displaying a significantly higher score (Figure 1A) (20). At the end of DSS treatment (day 7), CD300f/- mice displayed a shorter colon length (Figure 1B) and higher macroscopic inflammation scores (Figure 1C), and more severe disruption of the colonic architecture compared with $C D 300 f^{+/+}$mice (Figure 1D). It has not, to our knowledge, been previously reported that while the DAI score returned to normal in $\mathrm{CD}_{300 f^{+/+}}$mice, $\mathrm{CD} 300 \mathrm{f}^{-1-}$ mice maintained a high DAI score through 13 days following withdrawal of DSS (Figure 1A), indicating that CD300 $\mathrm{f}^{-/-}$mice failed to resolve colonic inflammation. In support, by day 14 , the colon length of $C D 300 f^{t /+}$ mice had returned to normal, whereas the colon lengths of $C D 300 f^{/-}$mice were shortened and similar to those from day 7 (Figure 1B). The macroscopic inflammation score and histological analysis also revealed a failure to resolve inflammation in CD300 $\mathrm{f}^{--}$mice (Figure 1, C and D).

To rule out differences in the gut microbiota as a cause of the increased disease severity observed in CD300f-deficient mice, we cohoused $C D 300 f^{7 /+}$ and $C D 300 f^{/-}$mice. Gut microbiome analysis revealed that the composition of microbes at the phylum level was comparable in these mice (Supplemental Figure 1A; supplemental material available online with this article; https://doi. org/10.1172/JCI89531DS1), indicating that the increased colitis severity in CD300f-deficient mice, still evident in these cohoused mice (Supplemental Figure 1, B-E), likely was not due to differences in the gut microbiota.

A hallmark of colitis is the infiltration of neutrophils into the colon. In agreement with disease severity, the number of neutrophils was higher in the lamina propria of $C D 300 f^{/-}$than $C D 300 f^{1 /+}$ mice during the inflammatory phase (Supplemental Figure 2A). The analysis of cell populations during the resolution phase (day 14) demonstrated a decrease in $C D 45^{+}$cells and neutrophil populations in the lamina propria of $C D 300 f^{+/+}$, but not $C D 300 f^{-/}$, mice, relative to the acute phase (day 7) (Supplemental Figure 2, A and $\mathrm{B}$ ), indicating a sustained recruitment or persistence of these cells in the inflamed colons of $C D 300 \mathrm{f}^{/-}$animals.

Taken together, our results show that the inflammation in $\mathrm{CD}_{300 \mathrm{f}^{/+}}$mice subsided after DSS withdrawal, whereas $C D 300 f^{/-}$mice exhibited a state of elevated and prolonged inflammation, thereby identifying CD300f as a key regulator of chronic inflammation in the gut.

CD300f deficiency leads to sustained and elevated TNF- $\alpha$ production by DCs, and IFN- $\gamma$ overproduction in the colon tissue. Having observed differences in the severity and resolution of colitis between $C D 30 O f^{+/+}$and $C D 30 O f^{/-}$mice, we next investigated whether they were related to differences in cytokine secretion. Analysis of cytokine levels in colon tissue lysates revealed that DSS challenge induced the production of TNF- $\alpha$, IFN- $\gamma$, IL- 6 , and IL-10 during the inflammation phase (day 7) in both $C D 300 f^{f^{/+}}$ and $C D 300 f^{/-}$mice, with $C D 300 f^{/-}$mice showing an increased production of TNF- $\alpha$ compared with $C D 30 O f^{+/+}$mice (Figure $2 \mathrm{~A}$ ).
Remarkably, while the levels of TNF- $\alpha$, IFN- $\gamma$, IL-6, and IL-10 returned to normal in the colons of $\mathrm{CD} 00 \mathrm{f}^{\mathrm{t}+}$ mice during the resolution phase (day 14), in the CD300f/- mice the TNF- $\alpha$ levels remained elevated, and the IFN- $\gamma$ and IL- 6 levels noticeably increased (Figure 2A). This indicates that prolonged and excessive production of proinflammatory cytokines is associated with a failure to resolve colonic inflammation. In agreement, immunofluorescence analysis revealed elevated levels of TNF- $\alpha$ in the colonic lamina propria of $\mathrm{CD} 0 \mathrm{Of}^{/-}$mice during both the initial inflammatory stage and the following resolution phase (Figure $2 \mathrm{~B}$ and Supplemental Figure 3A). Furthermore, the production of IFN- $\gamma$ in the colons of $C D 300 f^{/-}$but not $\mathrm{CD} 00 \mathrm{f}^{4 /+}$ mice was elevated during the resolution phase of colonic inflammation (Figure $2 \mathrm{~B}$ and Supplemental Figure 3B).

Since TNF- $\alpha$ was upregulated in the inflammatory phase of colitis in $C D 300 f^{/-}$mice, and IFN- $\gamma$ overproduction was observed

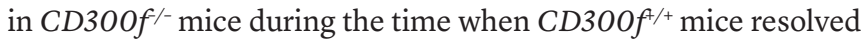
colonic inflammation (Figure 2A), we hypothesized that the excessive and prolonged production of TNF- $\alpha$ could promote IFN- $\gamma$ production by cells in the gut mucosa of $C D 300 f^{--}$mice, leading to failure to resolve gut inflammation. In agreement, $C D 300 f^{-1}$ mice treated with a TNF- $\alpha$-neutralizing antibody, infliximab (5), showed decreased susceptibility to colitis and better resolution of inflammation, as indicated by lower DAI scores (Figure 2C), longer colon lengths (Figure 2D), and decreased macroscopic (Figure 2E) and microscopic scores (Figure 2F), resembling values observed in $C D 300 f^{7 /+}$ mice. Following anti-TNF- $\alpha$ antibody treatment, the level of TNF- $\alpha$ in colon tissues was markedly decreased (Figure $2 G$ ), in line with previous reports showing reduction of TNF- $\alpha$ in sera or tissues of infliximab-treated animals $(21,22)$. Furthermore, infliximab treatment ameliorated production of IFN- $\gamma$, as well as IL-6, in CD300f/- mice (Figure $2 \mathrm{G}$ ), strongly supporting the notion that TNF- $\alpha$ leads to increased production of IFN- $\gamma$, and other proinflammatory cytokines. Unlike CD300 $f^{/-}$mice, antiTNF- $\alpha$ antibody treatment had a minimal impact on the severity of colitis or cytokine secretion in $\mathrm{CD} 00 \mathrm{f}^{\mathrm{f} /+}$ mice (Figure $2, \mathrm{C}-\mathrm{G}$ ).

Since the proinflammatory cytokines TNF- $\alpha$ and IFN- $\gamma$ have been shown to exacerbate intestinal inflammation (23), we investigated which populations of lamina propria cells were responsible for the elevated and continued TNF- $\alpha$ and IFN- $\gamma$ production observed in CD300 $f^{/-}$mice. We observed an enhanced number of TNF- $\alpha$-producing DCs at the onset of inflammation (day 4) in CD300f-deficient mice (Supplemental Figure 4). At day 7, lamina propria DCs were the most prominent population that showed a significant increase in both the percentage and the number of TNF- $\alpha$-producing cells in CD30Of/- mice compared with $\mathrm{CD} 300 \mathrm{f}^{+/+}$mice (Figure 3A and Supplemental Figure 5), suggesting that CD300f-deficient DCs are involved in enhanced production of TNF- $\alpha$ in the colon of $\mathrm{CD} 30 \mathrm{Of}^{/-}$mice during the inflammatory phase. Other cell populations, including macrophages, mast cells, and $\mathrm{T}$ cells, contributed to the sustained TNF- $\alpha$ overproduction in $C D 300 f^{/-}$mice over time (day 14; Figure 3B and Supplemental Figure 5); however, DCs had the biggest percentage of TNF- $\alpha-$ producing cells (Supplemental Figure 5), suggesting that DCs play a key role in this process.

Among DC subpopulations, CD $8 \alpha^{+}$conventional DCs and plasmacytoid DCs showed increased TNF- $\alpha$ production in 

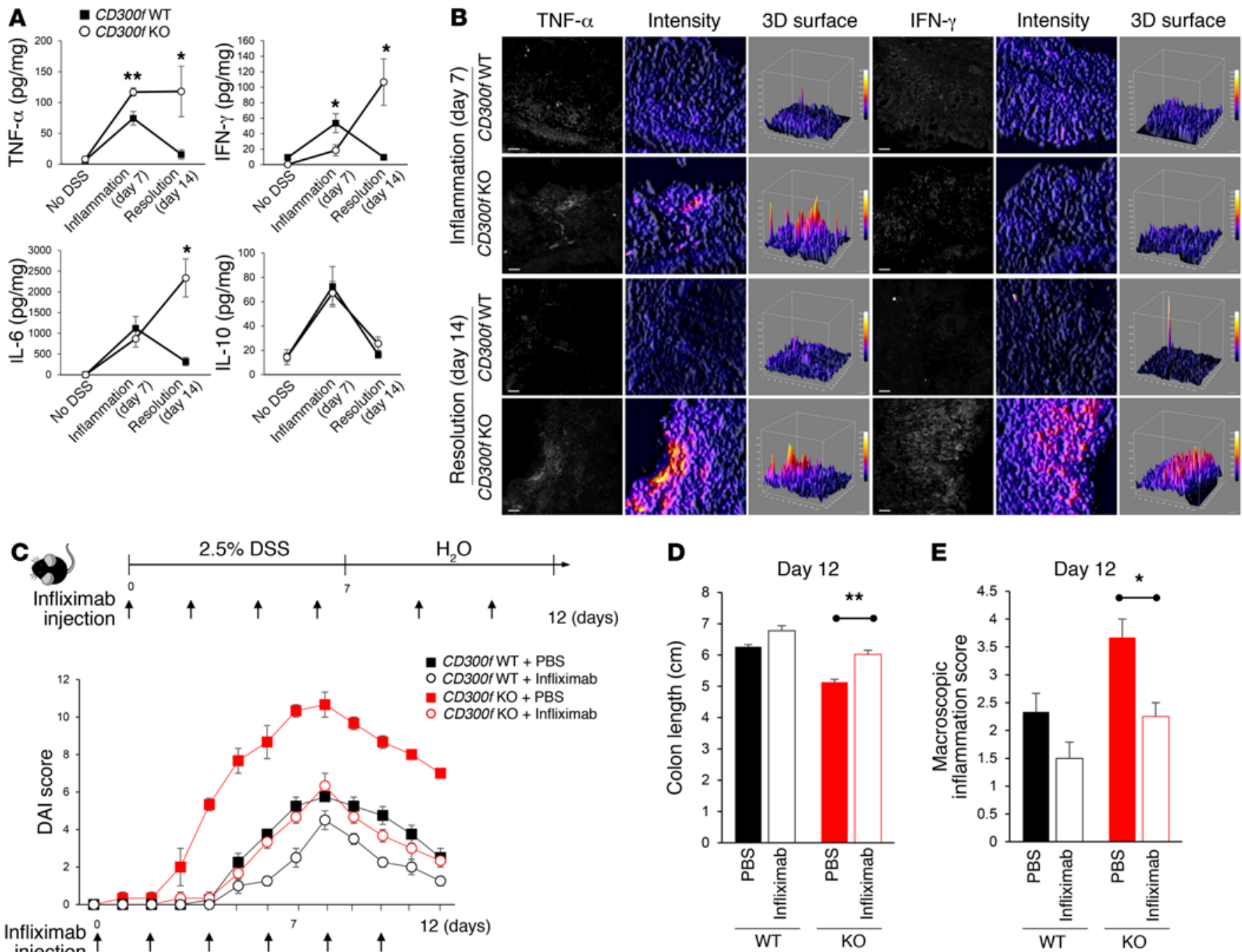
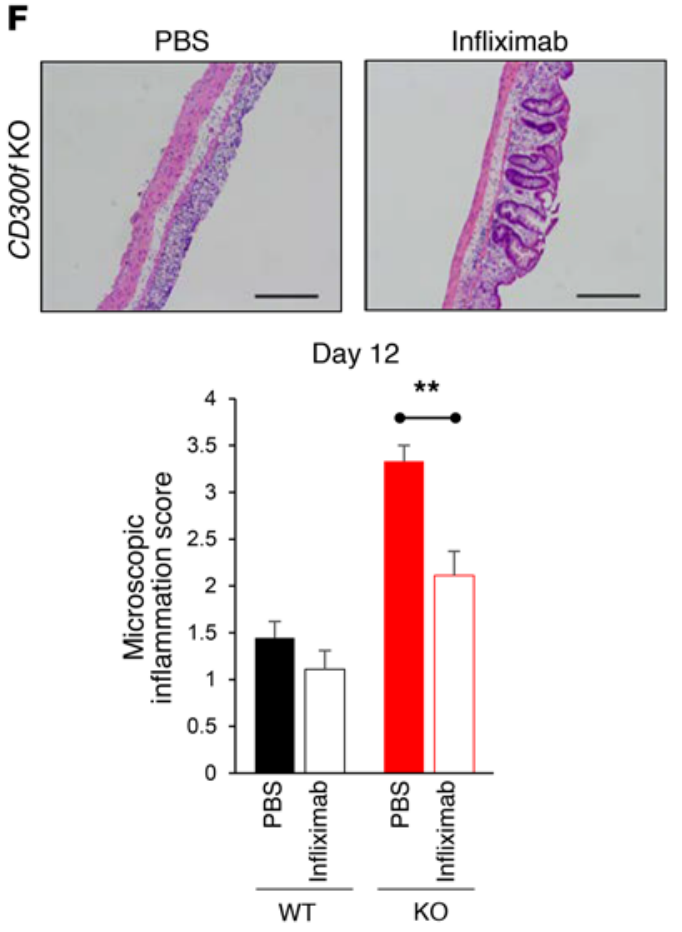

G
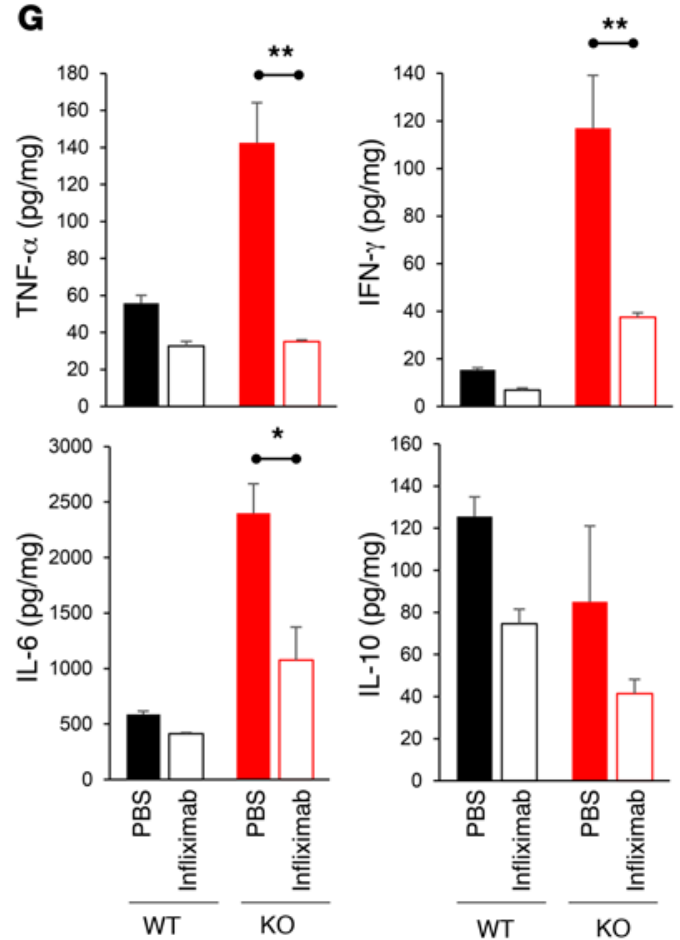
Figure 2. CD300f deficiency leads to sustained and elevated TNF- $\alpha$ production, which is responsible for induction of other proinflammatory cytokines and exacerbation of colonic inflammation. (A) Mice were treated as indicated in Figure $1 \mathrm{~A}$. Cytokine levels in the colon tissues of $C D 300 \mathrm{f}^{+/+}$and $C D 300 \mathrm{f}^{-/-}$ mice were determined on day 7 (Inflammation) and day 14 (Resolution). (B) Colon sections from CD300f//+ and CD300f $/$ - mice, collected on day 7 (Inflammation) or day 14 (Resolution), were stained for TNF- $\alpha$ or IFN- $\gamma$ (left panels); scale bars: $20 \mu \mathrm{m}$. The fluorescence signal intensity was determined for each

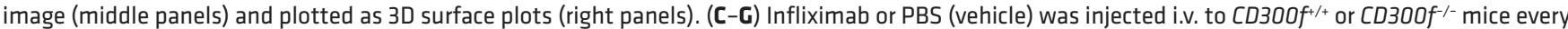
other day during 2.5\% DSS treatment, and every 3 days during the following 5 days. DAI was scored during and after DSS administration; infliximab injection times are indicated (C). On day 12, the colon length (D), macroscopic inflammation score (E), and microscopic inflammation score (F) were determined. The images in $\mathbf{F}$ illustrate representative results of H\&E staining of the colon tissues from CD300f $f^{-1}$ mice; scale bars: $500 \mu \mathrm{m}$. (C) Levels of the indicated cytokines in colon tissue lysates from $C D 300 \mathrm{f}^{+/+}$and $C D 300 \mathrm{f}^{-/-}$mice. All graphs show mean values $\pm \mathrm{SEM}$ ( $n=5$, each group). Two-tailed paired Student's $t$ test was used to determine statistical significance $\left({ }^{*} P<0.05,{ }^{* *} P<0.01\right)$.

$C D 300 f^{\prime-}$ mice during the inflammatory phase (day 7), whereas during the resolution phase (day 14) both $\mathrm{CD} 8 \alpha^{+}$conventional DCs and CD11 $\mathrm{b}^{+}$conventional DCs displayed elevated TNF- $\alpha$ production in $C D 300 f^{\prime-}$ mice (Supplemental Figure 6). There was no difference in the percentages of IFN- $\gamma$-producing cells in the colons

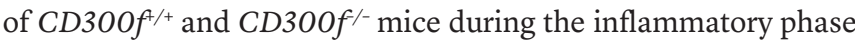
(Figure 3C). However, on day 14, there was a significant increase in the numbers and percentages of IFN- $\gamma$-producing T cells and NKT cells in the colons of CD3OOf/- compared with $C D 300 f^{1 /+}$ mice, with most of the IFN- $\gamma$ produced by $\mathrm{T}$ cells (Figure 3D). CD $8^{+} \mathrm{T}$ cells appeared to be the main producers of IFN- $\gamma$ among different $\mathrm{T}$ cell populations, and $\mathrm{CD} 8^{+} \mathrm{T}$ cells isolated from lamina propria of $C D 300 f^{\prime}-$ mice displayed distinctly increased IFN- $\gamma$ production when compared with $C D 300 f^{t /+}$ animals (Supplemental Figure 7). Since $T$ cells do not express CD300f (18), these results suggest that the IFN- $\gamma$ overproduction is likely an indirect and secondary effect of $\mathrm{CD} 300 \mathrm{f}$ deficiency that, nonetheless, contributes to impaired resolution of colitis in $C D 300 f^{\prime-}$ mice.

PS-mediated efferocytosis by CD300f-deficient DCs is responsible for elevated TNF- $\alpha$ production and exacerbation of colonic inflammation. As CD300f functions in recognition and clearance of ACs $(16,17)$, and prompt efferocytosis is critical for resolution of inflammation and tissue repair (24), we next investigated AC accumulation in inflamed colon tissues. Compared with $\mathrm{CD} 300 \mathrm{f}^{1 /+}$ mice, we observed a markedly increased number of TUNEL ${ }^{+} \mathrm{ACs}$ in the lamina propria of $C D 300 f^{/-}$mice during both the inflammation and resolution phases of colitis (Figure 4A). We found that CD300f-deficient macrophages were most likely responsible for impaired clearance of ACs (18) generated during inflammation in CD300 $f^{\prime-}$ mice. Transfer of CFSE-labeled CD3OOf/+ bone marrow-derived macrophages (BMM $\phi$; Supplemental Figure 8) into

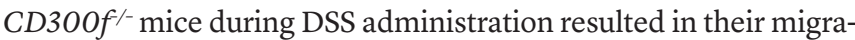
tion to the inflamed colonic tissue (Supplemental Figure 9A), and a decrease of TUNEL ${ }^{+} \mathrm{ACs}$ in the lamina propria of $C D 300 f^{-}$- mice (Supplemental Figure 9B). This correlated with the fact that transferred $C D 300 f^{f^{\prime+}} \mathrm{BMM} \phi$, but not $C D 300 f^{/-} \mathrm{BMM} \phi$, attenuated the susceptibility to colitis, as indicated by DAI score, colon length, and macroscopic and microscopic inflammation scores (Supplemental Figure 9, C-F), and showed a more active uptake of apoptotic neutrophils (Supplemental Figure 9, G-I) that commonly appear during colonic inflammation (25). These findings suggest that an elevated accumulation of ACs, resulting from impaired efferocytosis by CD300f-deficient macrophages, contributes to the excessive and prolonged colonic inflammation in $C D 300 f^{\prime-}$ mice.

Since DCs were the major population displaying altered TNF- $\alpha$ production in the inflamed colon, we next investigated whether DSS or ACs could affect TNF- $\alpha$ production by DCs. We found that exposure to DSS did not induce cytokine production by purified bone marrow-derived DCs (BMDCs) from either $\mathrm{CD} 300 \mathrm{f}^{\mathrm{f} /+}$ or CD30Of/- mice (Supplemental Figure 10). To determine whether efferocytosis promoted TNF- $\alpha$ production by DCs, purified BMDCs isolated from $C D 300 f^{\prime /+}$ and $C D 300 f^{/-}$mice were incubated with apoptotic thymocytes. While DCs from both strains could engulf ACs, DCs from CD300f-deficient mice were twice as efficient (Figure $4 \mathrm{~B})$. Importantly, TNF- $\alpha$ production was significantly elevated in CD300f-deficient BMDCs stimulated with ACs, whereas no induction of TNF- $\alpha$ production was observed for CD30Of ${ }^{\prime /+}$ BMDCs (Figure 4C). Efferocytosis did not induce IFN- $\gamma$ production in either $C D 300 f^{/ /+}$or $C D 300 f^{/-}$BMDCs (Figure $4 \mathrm{C}$ ). Uptake of ACs by BMM $\phi$ from either $C D 300 \mathrm{f}^{\prime /+}$ or $C D 300 \mathrm{f}^{/ /}$mice did not alter the amounts of TNF- $\alpha$ and IFN- $\gamma$ produced by these cells, but upregulated IL-10 production by $\mathrm{CD} 300 \mathrm{f}^{\prime /+} \mathrm{BMM} \phi$ (Figure $4 \mathrm{C}$ ), consistent with previous data (26) and the fact that they are more efficient at engulfing ACs than $C D 300 f^{\prime-} \mathrm{BMM} \phi(17)$. Efferocytosis did not induce IL-10 production by $C D 300 f^{/ /+}$BMDCs, but induced a slight increase in IL-10 production by $C D 300 f^{/}-$BMDCs (Figure 4C). However, efferocytosis-induced IL-10 production is apparently insufficient to balance the inflammatory effects resulting from the elevated TNF- $\alpha$ production by CD300f-deficient DCs engulfing ACs, which agrees with the observation that elevated levels of IL-10 are often present in patients with IBDs (27).

To confirm that the enhanced TNF- $\alpha$ production by CD300 $f^{\wedge}$ BMDCs was due to PS-mediated engulfment of ACs, CD300f $/$ BMDCs were preincubated with PS-containing liposomes before the addition of ACs. PS, but not phosphatidylcholine $(\mathrm{PC})$, liposomes attenuated the effect of AC stimulation on TNF- $\alpha$ production by CD30O $f^{\prime-}$ BMDCs, but alone did not affect TNF- $\alpha$ production (Figure 4D), indicating that engulfment of ACs, not just PS recognition, is required to stimulate TNF- $\alpha$ production. Finally, to verify that the observed difference in cytokine production was related to AC uptake rather than bacterial phagocytosis, both of which occur in the inflamed colon tissue, we coincubated the BMDCs with E. coli. There was no difference in the amount of the phagocytosed bacteria or TNF- $\alpha$ production between $C D 300 f^{1 /+}$ and $C D 300 f^{/-}$BMDCs (data not shown), indicating that phagocytosis of bacteria is not a factor determining the difference in DC-mediated TNF- $\alpha$ production between $\mathrm{CD} \mathrm{OOO \textrm {f } ^ { \prime / + }}$ and $\mathrm{CD} 30 \mathrm{O} \mathrm{f}^{-/}$mice. Because IFN- $\gamma$ production was mainly observed in lamina propria $\mathrm{T}$ cells (Figure 3D), and $\mathrm{T}$ cells do not express CD300f (18), we next investigated whether $\mathrm{T}$ cells produced IFN- $\gamma$ in response to TNF- $\alpha$ secreted from efferocytic DCs. Incubation of T cells with TNF- $\alpha-$ containing medium from a culture of purified $C D 300 f^{\prime-}$ BMDCs that had engulfed ACs, or medium supplemented with TNF- $\alpha$, 
A
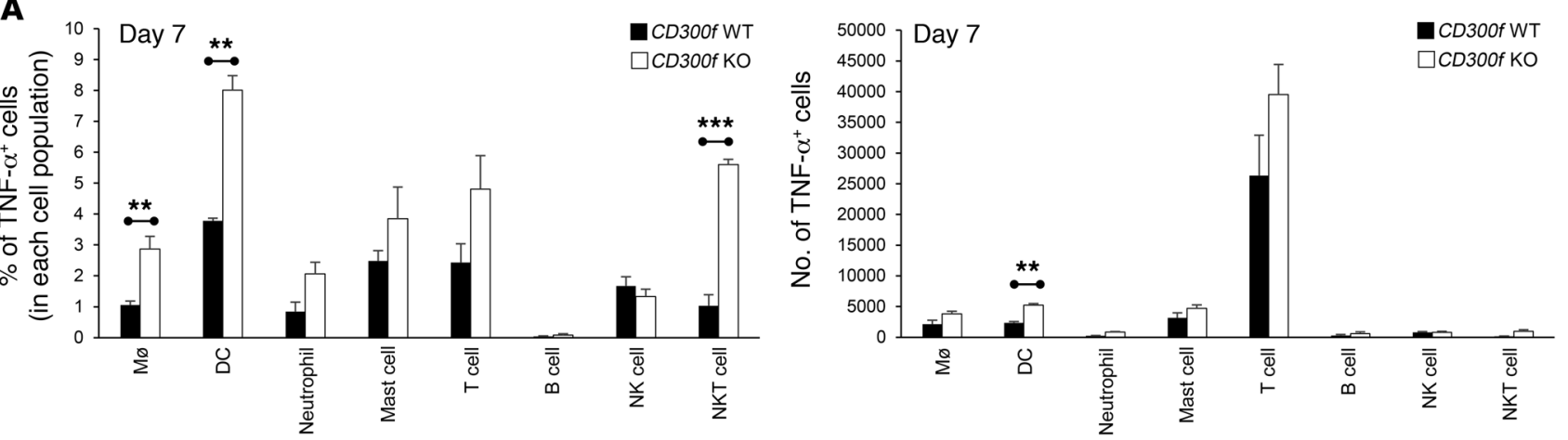

B
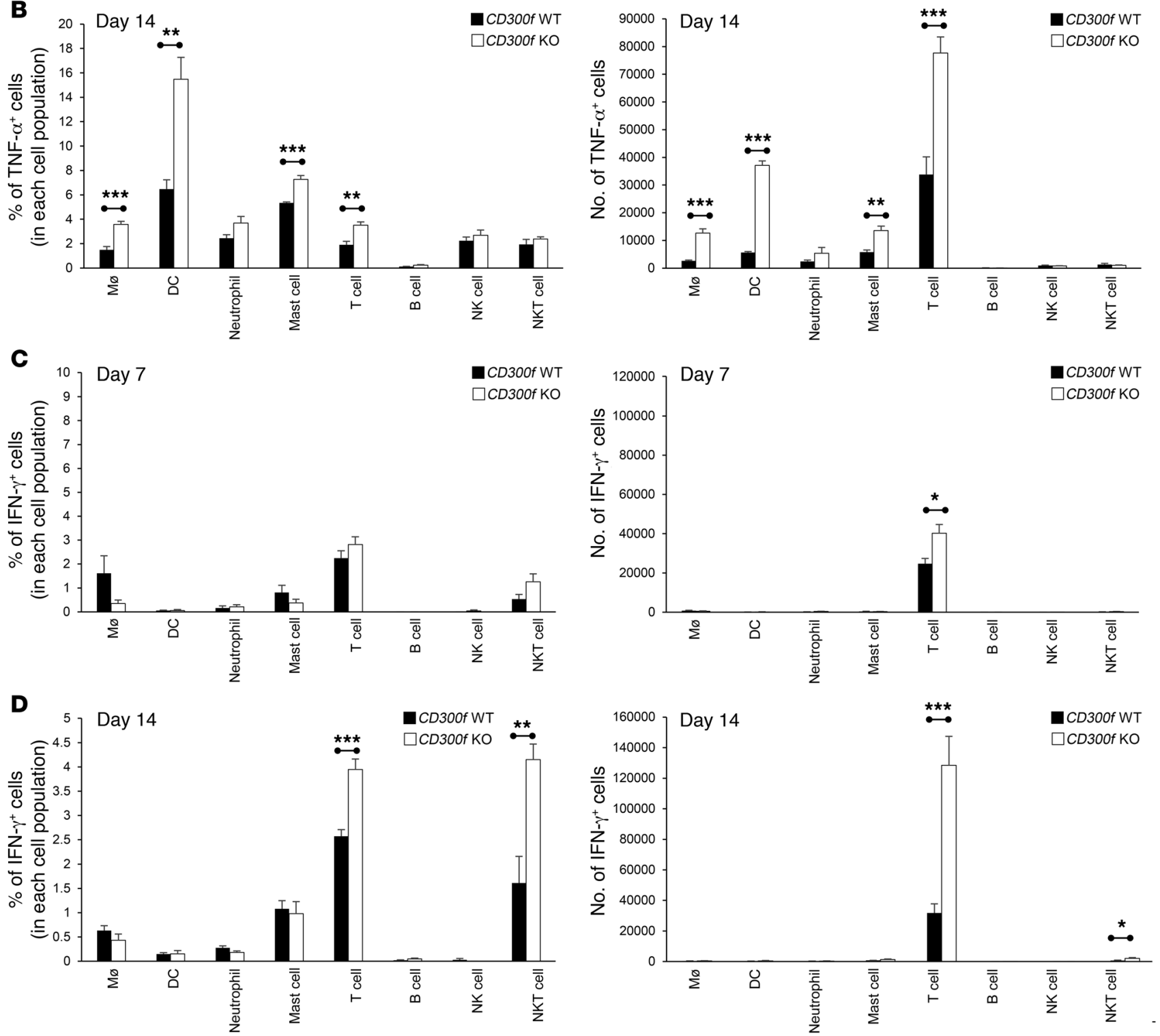

Figure 3. Identification of cells producing TNF- $\alpha$ and IFN- $\gamma$ in the inflamed colon tissue. Lamina propria cells were isolated from the colons of DSStreated $C D 300 \mathrm{f}^{+/+}$or $C D 300 f^{-/-}$mice, collected on day 7 (A and $\mathbf{C}$ ) or day 14 (B and $\left.\mathbf{D}\right)$. The intracellular expression of TNF- $\alpha$ and IFN- $\gamma$ was determined by

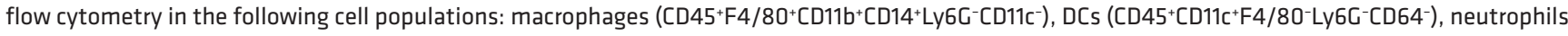
(CD45+CD11b+Ly6C+CD11C-F4/80'), mast cells (CD45+CD11b+FceRI+), T cells (CD45+CD3+), B cells (CD45+CD3-CD19+), NK cells (CD45+CD3-NK1.1+), and NKT cells (CD45+CD3+NK1.1+). The graphs show the percentages (left) and total numbers (right) of cells expressing TNF- $\alpha(\mathbf{A}$ and $\mathbf{B})$ or IFN- $\gamma(\mathbf{C}$ and $\mathbf{D})$. Data are expressed as means + SEM ( $n=3-8$, each group). Two-tailed paired Student's $t$ test was used to determine statistical significance $\left({ }^{*} P<0.05,{ }^{* *} P<0.01\right.$, $\left.{ }^{* * *} P<0.001\right)$. 
resulted in increased production of IFN- $\gamma$ (Figure 4E). Furthermore, antibody-mediated neutralization of TNF- $\alpha$ ameliorated IFN- $\gamma$ production by $\mathrm{T}$ cells (Figure $4 \mathrm{E}$ ), indicating that TNF- $\alpha$ is an inducer of IFN- $\gamma$ production.

Taken together, our results show that CD300f deficiency enhances efferocytosis by DCs and leads to elevated production of TNF- $\alpha$, a key cytokine aggravating DSS-induced colitis in CD300f ${ }^{/-}$mice.

CD300f-deficient DCs exacerbate colonic inflammation. To verify the importance of CD300f-deficient DCs in colitis pathogenesis, we transferred purified $C D 300 f^{+/+}$or $C D 300 f^{/-}$BMDCs (Supplemental Figure 8) into $C D 300 f^{+/+}$mice on day 4 of DSS administration (Figure 5A). Within 2 days after transfer, $C D 300 f^{\prime-}$ BMDCs, but not $C D 300 f^{7 /+}$ BMDCs, markedly increased disease severity in the $C D 300 f^{/ /+}$recipient mice as indicated by the DAI score (Figure $5 \mathrm{~B}$ ). On day 7 , there was no significant difference in colon length (Figure 5C) and macroscopic inflammation scores (Figure 5D) between mice receiving $\mathrm{CD} 00 \mathrm{f}^{\mathrm{t}+}$ and those receiving $C D 300 f^{1-}$ BMDCs. However, histological analyses revealed a massive infiltration of inflammatory cells and disruption of colonic structure in the epithelium and lamina propria of mice receiving $C D 300 f^{1-}$ BMDCs (Figure $5 \mathrm{E}$ ). By day 14, the greater severity of the DSS-induced colitis in mice receiving CD3OOf $\mathrm{f}^{-\mathrm{B}} \mathrm{BMDC}$ was also associated with shortened colon lengths (Figure $5 \mathrm{C}$ ) and increased macroscopic (Figure 5D) and microscopic inflammation scores (Figure 5E). To investigate how the transferred CD300f/BMDCs exacerbated colitis, we analyzed cytokine profiles in the colon tissue of the recipient mice. Compared with $C D 300 f^{t /+}$, $\mathrm{CD} 00 \mathrm{f}^{/-} \mathrm{BMDC}$ transfer induced significant upregulation of TNF- $\alpha$, IFN- $\gamma$, and IL- 6 production in the colon during the inflammatory phase (day 7; Figure 5F); the enhanced production of IFN- $\gamma$ was evident well after DSS withdrawal (day 14; Figure 5F). Collectively, these results demonstrate that CD300f-mediated regulation of DC activity is responsible for the enhanced production of proinflammatory cytokines, and plays a major role in the increased severity of colonic inflammation in CD30Of/- mice.

\section{Discussion}

Aberrant clearance of ACs in the intestinal lamina propria has been implicated as one of the primary reasons for failure to resolve inflammation in IBDs $(28,29)$. DSS-induced colitis, a mouse model mimicking characteristics of human IBD, has been used to investigate this complex multifactorial disease (19). It is generally thought that DSS induces epithelial cell death, thereby disrupting the intestinal epithelial barrier, leading to infiltration of gut microorganisms into the mucosa (19). This activates tissue-resident macrophages and DCs to recruit neutrophils, which transiently elevate inflammatory responses in order to defend the host against microbial assault $(30,31)$. After performing their antimicrobial functions, neutrophils undergo apoptosis, and are subsequently removed by phagocytes, mainly macrophages (32, 33), promoting resolution of acute inflammation and a return to homeostasis (25). We have reported that the recognition of PS on ACs by CD300f mediates efferocytosis by phagocytes (16-18). Given this, we hypothesized that regulation of efferocytosis through CD300f might be involved in controlling inflammation in the gut. We found that compared with $C D 300 \mathrm{f}^{\mathrm{f}^{/+}}$mice,
$\mathrm{CD}_{0} 0 \mathrm{f}^{\mathrm{A}}$ - mice were more prone to acute intestinal inflammation, in line with a previous report (20). Our findings show that CD300f expression plays a positive role in limiting gut inflammation. This is in contrast to a recent report by Moshkovits et al. demonstrating that CD300f expression promotes colonic inflammation, a function they associated with colonic eosinophils (34). We can only speculate as to the reason for the opposite results. For instance, Moshkovits et al. did not detect CD300f expression on colonic macrophages or mast cells, and they failed to examine CD300f expression on DCs (34), whereas we detect CD300f expression on macrophages, DCs, neutrophils, and mast cells in colonic lamina propria (Supplemental Figure 11); similarly, Matsukawa et al. (20) readily detected CD300f on the surface of colonic mast cells, macrophages, and eosinophils. The lack of detection of CD300f on lamina propria macrophages by Moshkovits and colleagues is surprising, as, in general, macrophages could be considered a hallmark of CD300f expression (18, 35-37). CD300f expression has been shown to be regulated by various cytokines $(38,39)$, which could depend on exposure to environmental factors and/ or different microbiota communities present in the animal facilities. Although cohousing revealed that the increased sensitivity of colitis in CD300f-deficient mice was not due to differences in the gut microbiota in our experimental settings, we cannot exclude the possibility of substantial differences in the composition of gut microbiota or other environmental factors across the different animal facilities used in all 3 studies.

Importantly, we discovered that $C D 300 f^{/-}$mice failed to resolve inflammation in a timely manner, a previously unrecognized finding that is highly relevant to human condition, as IBDs are chronic inflammatory disorders. Associated with this failure to resolve colonic inflammation and increased disease severity, we found that dysregulation of DC efferocytosis can cause sustained gut inflammation. Enhanced DC efferocytosis, due to the lack of CD300f, induced DCs to overproduce TNF- $\alpha$, which stimulated the production of additional proinflammatory cytokines, particularly IFN- $\gamma$, by lamina propria T cells. Accumulation of activated DCs has been observed at the inflamed sites in colons of human IBD patients and mice with experimentally induced colitis (40), with no clear indication as to their role in the disease pathogenesis $(30,41)$. Our data indicate that dysregulation of DC efferocytosis may contribute to chronic gut inflammation.

A search through the Catalog of Published Genome-Wide Association Studies (http://www.genome.gov/gwastudies), a database of SNP-trait associations extracted from published GWAS, reveals several CD300f SNPs (rs34303409, rs35489971, rs749780, rs1037170, rs9906320) associated with ulcerative colitis. Whether CD300f is dysregulated on myeloid cells of IBD patients remains to be determined.

It is important to note that others have reported the involvement of other PS receptors in the acute phase of experimental colitis. MFG-E8, a soluble PS-receptor bridging molecule that promotes efferocytosis, is known to have a protective effect on gut epithelial homeostasis, and its deficiency in macrophages results in increased susceptibility to colitis and aberrant mucosal healing $(42,43)$. Combined loss of AXL and MER expression impairs the clearance of ACs by macrophages, thereby increasing susceptibility to colitis and inflammation-associated colorectal cancer (44). 
A

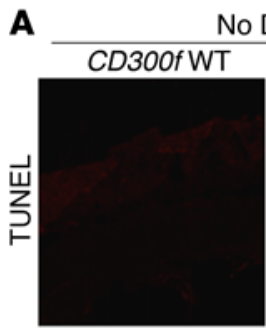

No DSS
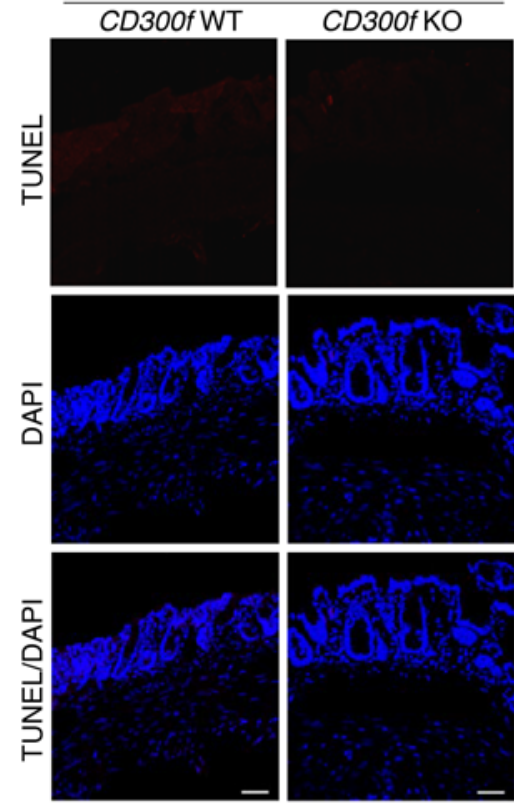

B
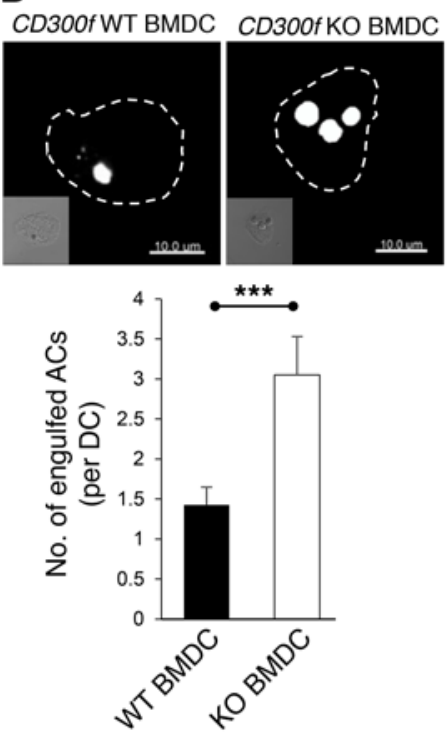

D

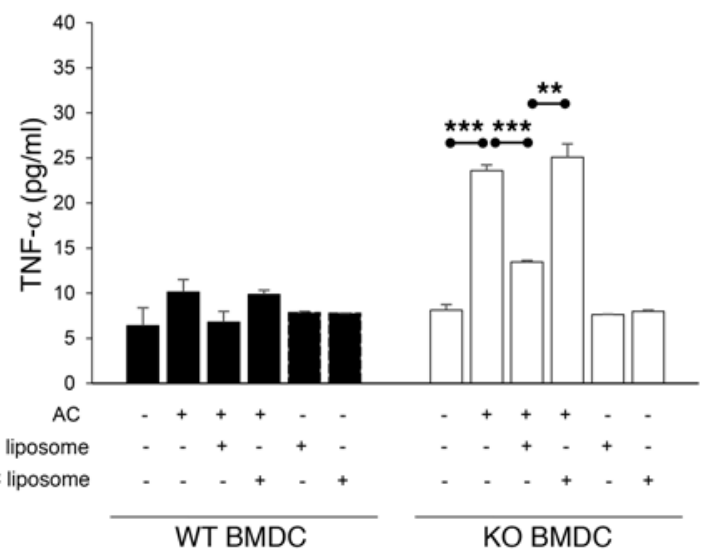

C
Inflammation (day 7)
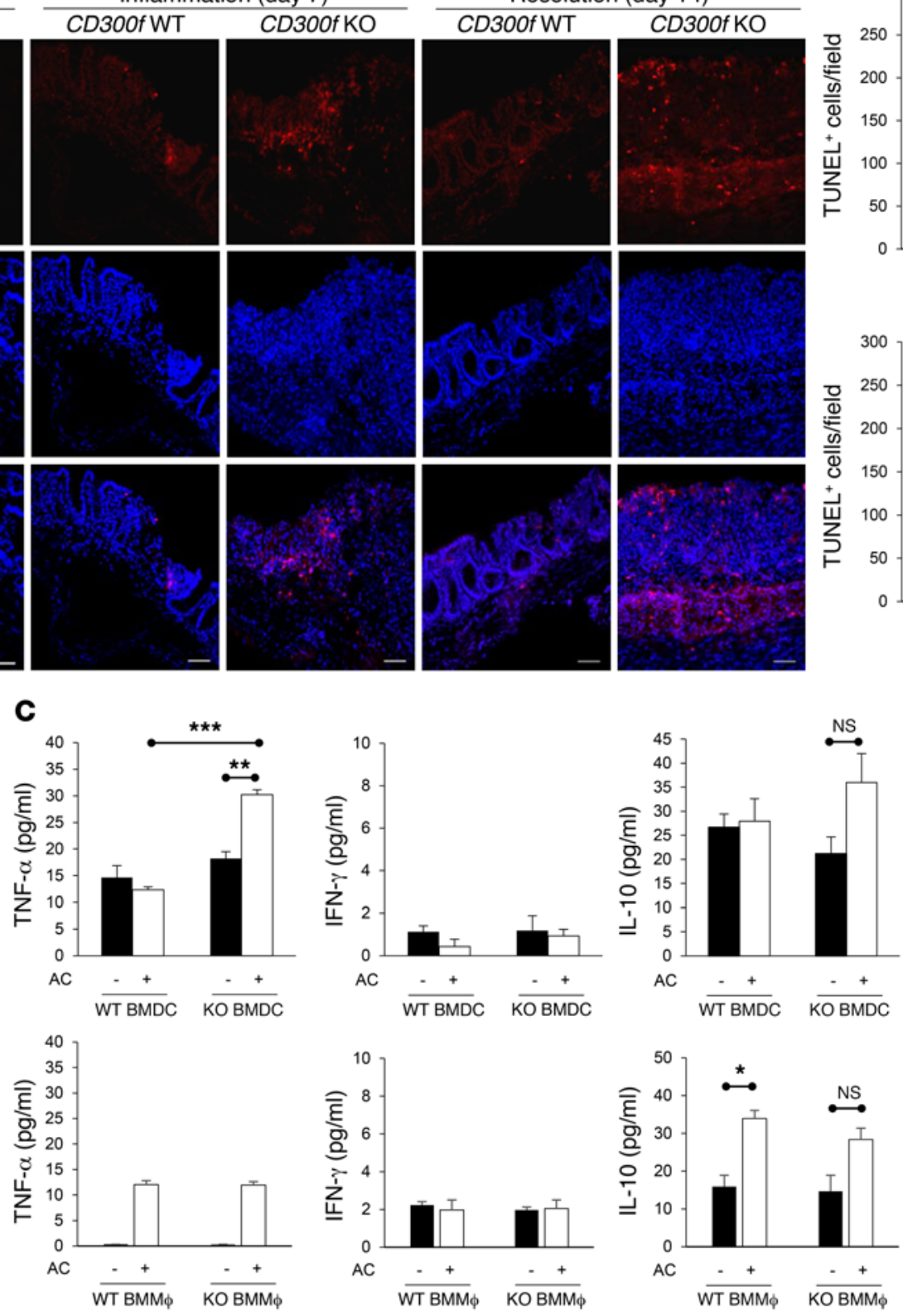

E

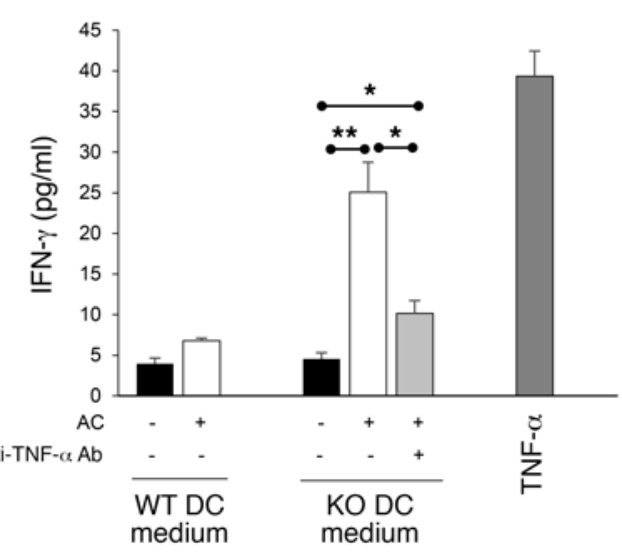

Resolution (day 14)
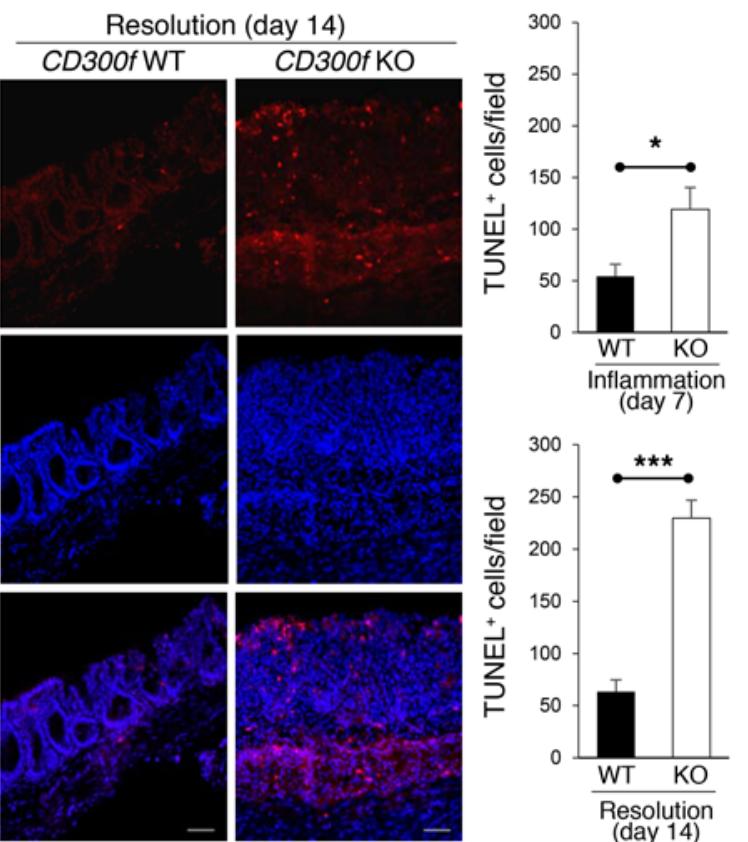

(day 14) 
Figure 4. PS-mediated engulfment of ACs by CD300f-deficient DCs induces their TNF- $\alpha$ production, and stimulates IFN- $\gamma$ secretion by T cells. (A) The presence of ACs in colons was assessed by TUNEL staining. The images show representative immunofluorescence staining for TUNEL (red) in colon sections from $C D 300 \mathrm{f}^{+/}$and $C D 300 \mathrm{f}^{-/-}$mice. Nuclei were visualized with DAPI (blue). Scale bars: $50 \mu \mathrm{m}$. The graphs show the quantification of TUNEL ${ }^{+}$cells per field of view (3 fields per colon section). (B) $C D 300 \mathrm{f}^{+/+}$or $C D 300 \mathrm{f}^{-/-} \mathrm{BMDCs}$ were coincubated with pHrodo-labeled apoptotic thymocytes for 30 minutes, followed by confocal microscopy analysis. The images (top) show representative images of BMDCs with engulfed ACs (white); scale bars: $10 \mu \mathrm{m}$. The graph (bottom) shows the quantification of AC engulfment by BMDCs. (C-E) BMDCs or BMM $\phi$ from $C D 300 \mathrm{f}^{+/+}$or $C D 300 f^{-/-}$mice were coincubated with apoptotic thymocytes in the absence (C and $\mathbf{E}$ ) or the presence of PBS, PS:PC liposomes, or PC:PC liposomes (D) for 30 minutes, followed by incubation in a fresh medium for 48 hours. The cell culture media were collected and used to analyze the levels of the indicated cytokines (C and $\mathbf{D})$ or to culture splenic T cells for 48 hours in the presence or absence of anti-TNF- $\alpha$ antibodies (E). To calculate the concentration of IFN- $\gamma$ released from T cells, the amount of cytokines present in the conditioned media was subtracted from the final values. The media from T cells cultured for 48 hours in the presence of TNF- $\alpha$ ( 200 $\mathrm{ng} / \mathrm{ml}$ ) were used as a positive control (E). Data are expressed as means $+\mathrm{SEM}(n=15$, each group in $\mathbf{A} ; n=50$ cells analyzed in 3 independent experiments in $\mathbf{B} ; n=3$ in C-E). Two-tailed paired Student's $t$ test was used to determine statistical significance $\left({ }^{*} P<0.05,{ }^{* *} P<0.01,{ }^{* *} P<0.001\right)$.

Collectively, the combined data suggest that different PS receptors, for yet undefined reasons, are playing nonredundant roles in regulating macrophage efferocytosis.

Unlike other PS receptors, CD300f is a unique and intriguing member of the CD300 family, as it contains tyrosine-based motifs with either activating or inhibitory potential. Consequently, mouse CD300f has been shown to serve as either an activating or an inhibitory receptor $(17,18,45-48)$. Our previous work showed that the CD300f cytoplasmic tail initiates either positive or negative signals for phagocytosis of ACs upon recognition of PS (17). The positive signals promote phagocytosis of ACs through recruitment of the p85 regulatory subunit of PI3K, and activation of the PI3K-Rac/CDC42 pathway and filamentous actin rearrangements (17). The inhibitory signals, initiated by SHP-1 phosphatase recruitment to CD300f, serve to stop efferocytosis, and correlate with SHP-1 expression levels (17). Our data indicate that CD300f might function differently depending on the available signaling capacity within a specific cell type $(17,18)$. In macrophages, expression of $\mathrm{CD} 300$ f generates positive signals that dominate over negative signals (17); however, in DCs the inhibitory cues dominate, as CD300f-expressing DCs show decreased efferocytosis compared with CD300f-deficient DCs. A possible explanation for this dichotomy in function could be different availability of kinases and/or phosphatases in macrophages and DCs. According to information found in the Immunological Genome Project database (https://www.immgen.org), SHP-1 appears to be expressed at about 2-fold higher levels in DCs than in macrophages, while the $\mathrm{p} 85$ regulatory subunit of PI3K is expressed at an almost 2-fold lower level in DCs compared with macrophages, at least in the spleen. Thus, higher SHP-1 expression levels in DCs than in macrophages could lead to its more efficient recruitment by CD300f, resulting in inhibitory signaling in DCs. Our future studies will focus on detailing the mechanisms responsible for this contrasting function of CD300f in DCs and macrophages.

We propose that under normal physiological conditions, CD300f promotes the efficient clearance of ACs by macrophages, and suppresses DC-mediated efferocytosis and TNF- $\alpha$ production by DCs. Thus, the inflammation is quickly resolved, as ACs are efficiently phagocytized and the DC-initiated proinflammatory cytokine cascade is not triggered (Figure 6). However, under CD300f deficiency, the impaired macrophage-mediated efferocytosis leads to accumulation of ACs (including apoptotic neutrophils). This leads to enhanced exposure of apoptotic/necrotic cells to CD300f-deficient DCs, which ingest those ACs and subsequently produce TNF- $\alpha$, which could further inhibit macrophage efferocytosis (49), thereby additionally contributing to AC accumulation in the gut of $C D 300 f^{-}$- mice. Moreover, TNF- $\alpha$ stimulates $\mathrm{T}$ cells, and likely other surrounding cells, to produce IFN- $\gamma$ (Figure 6). TNF- $\alpha$ and IFN- $\gamma$ are known to synergistically induce intestinal barrier disruption, which exacerbates inflammation in the colon $(23,50)$. Thus, elevated production of TNF- $\alpha$ by DCs leading to enhanced IFN- $\gamma$ production in CD300f deficiency likely contributes to additional disturbance of the intestinal barrier, allowing more bacteria to enter, resulting in prolonged recruitment to and/or activation of neutrophils and other immune cells (e.g., mast cells) at the inflamed site $(20,51,52)$. This, in turn, could induce additional tissue damage through release of ROS, chemoattractants, and proinflammatory mediators $(20,53)$. Ultimately, a perpetual feedback loop of proinflammatory conditions is generated, leading to failure to resolve inflammation and, subsequently, development of chronic inflammation (Figure 6).

In conclusion, we show that physiological consequences of CD300f deficiency are DC-initiated proinflammatory cytokine overproduction and excessive inflammatory responses in the gut, stemming from an unbalanced clearance of ACs, leading to failure to resolve intestinal inflammation. Our findings highlight a previously unappreciated function of CD300f expression by professional phagocytes, especially DCs, in controlling the duration and severity of intestinal inflammation, and underscore the importance of CD300f-expressing myeloid cells in maintaining gut immune homeostasis.

\section{Methods}

Animals. CD30Of//+ and CD300f/- mice, generated by Ozgene (17), were bred and housed in a pathogen-free environment in the National Institute of Allergy and Infectious Diseases (NIAID) animal facility. In all in vivo experiments, 6- to 8-week-old male mice were used.

DSS-induced colitis. To induce colitis, CD300 $\mathrm{f}^{\mathrm{f} /}$ and CD30Of/mice were given drinking water containing 2.5\% DSS (MW 36,00050,000; MP Biomedicals) for 7 days. For evaluation of colitis resolution, mice were given normal drinking water for an additional 7 days after DSS treatment. For BMM $\phi$ or BMDC transfer experiments, mice were injected i.v. with $2 \times 10^{6}$ cells labeled with CFSE during DSS administration. For TNF- $\alpha$ neutralization experiments, infliximab (Janssen Biotech), previously validated in several mouse disease models $(5,21$, $22,54)$, was injected $(10 \mathrm{mg} / \mathrm{kg})$ into CD300f/- mice every other day during DSS treatment, and every 3 days during the following 7 days.

Inflammation assessment. DAI score was determined by daily assessment of weight loss, stool consistency, and rectal bleeding. Scores were defined as follows: weight loss was graded 0 for $<1 \%$ loss, 
A

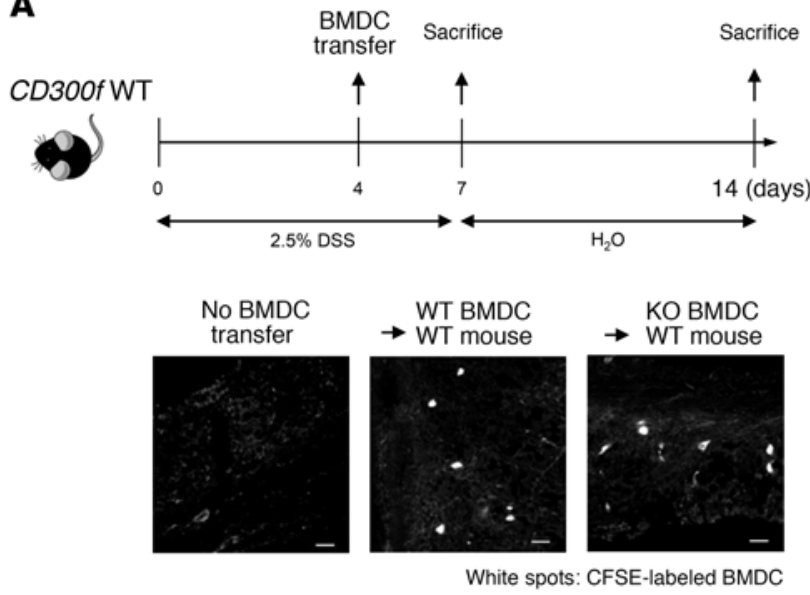

B



C

Inflammation (day 7)

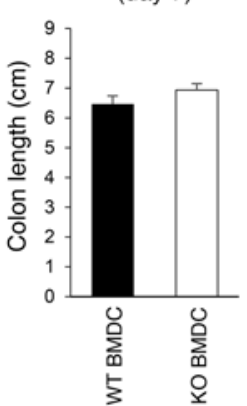

$\mathbf{F}$

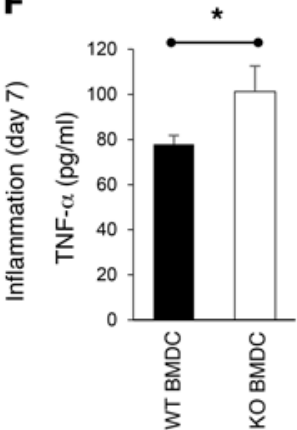

Resolution (day 14)

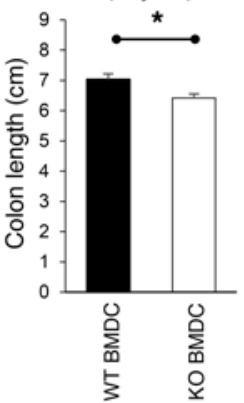

D

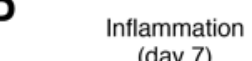

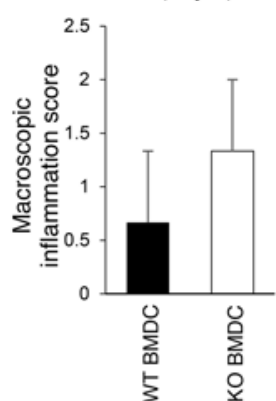

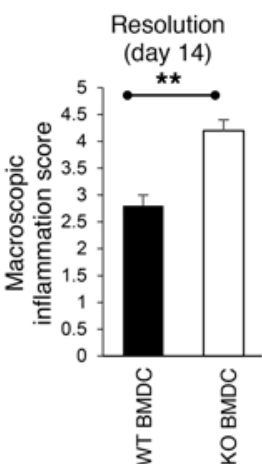

(day 14)

E
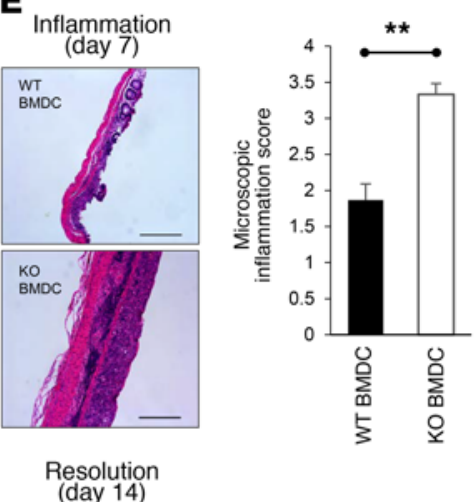

(day 14)
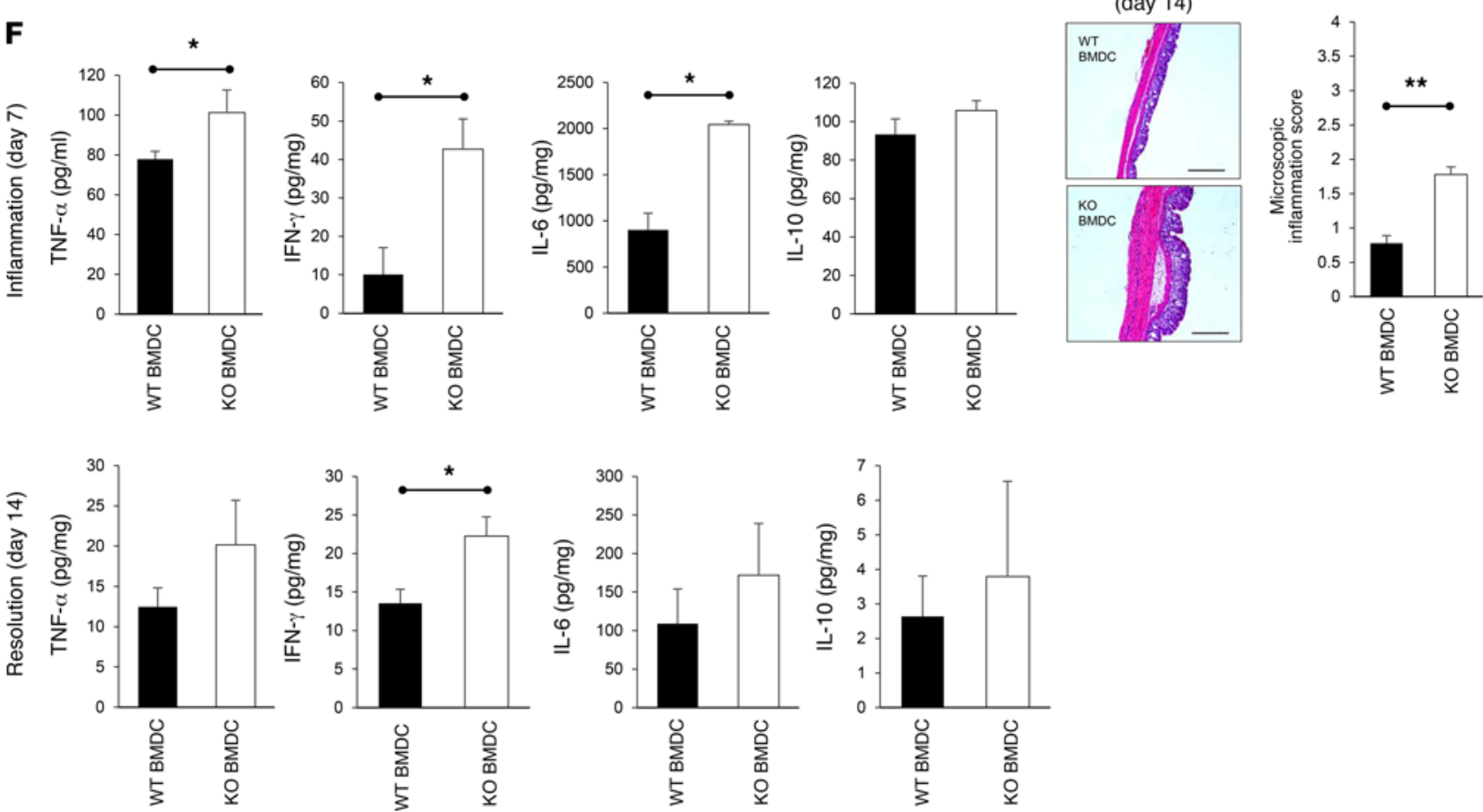

Figure 5. CD300f-deficient BMDCs exacerbate inflammation and delay resolution of inflammation in colonic inflammation. (A) Purified, CFSE-labeled

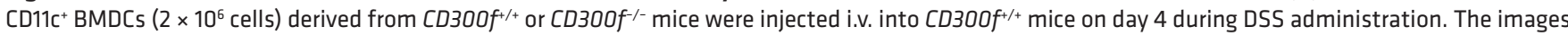
illustrate the presence of the transferred, CFSE ${ }^{+}$BMDCs in the colon tissues; scale bars: $20 \mu \mathrm{m}$. (B) DAI scored during and after DSS administration; BMDC transfer time is indicated. (C-E) The colon length (C), macroscopic inflammation score (D), and microscopic inflammation score (E) were determined on day 7 (Inflammation) and day 14 (Resolution). The images in E are representative images of H\&E-stained colon tissues; scale bars: $500 \mu$ m. (F) Colon tissue lysates were evaluated for the indicated cytokine levels. All graphs show mean values; error bars indicate SEM ( $n=4$, each group). Two-tailed paired Student's $t$ test was used to determine statistical significance $\left({ }^{*} P<0.05\right.$, $\left.{ }^{* *} P<0.01\right)$. 


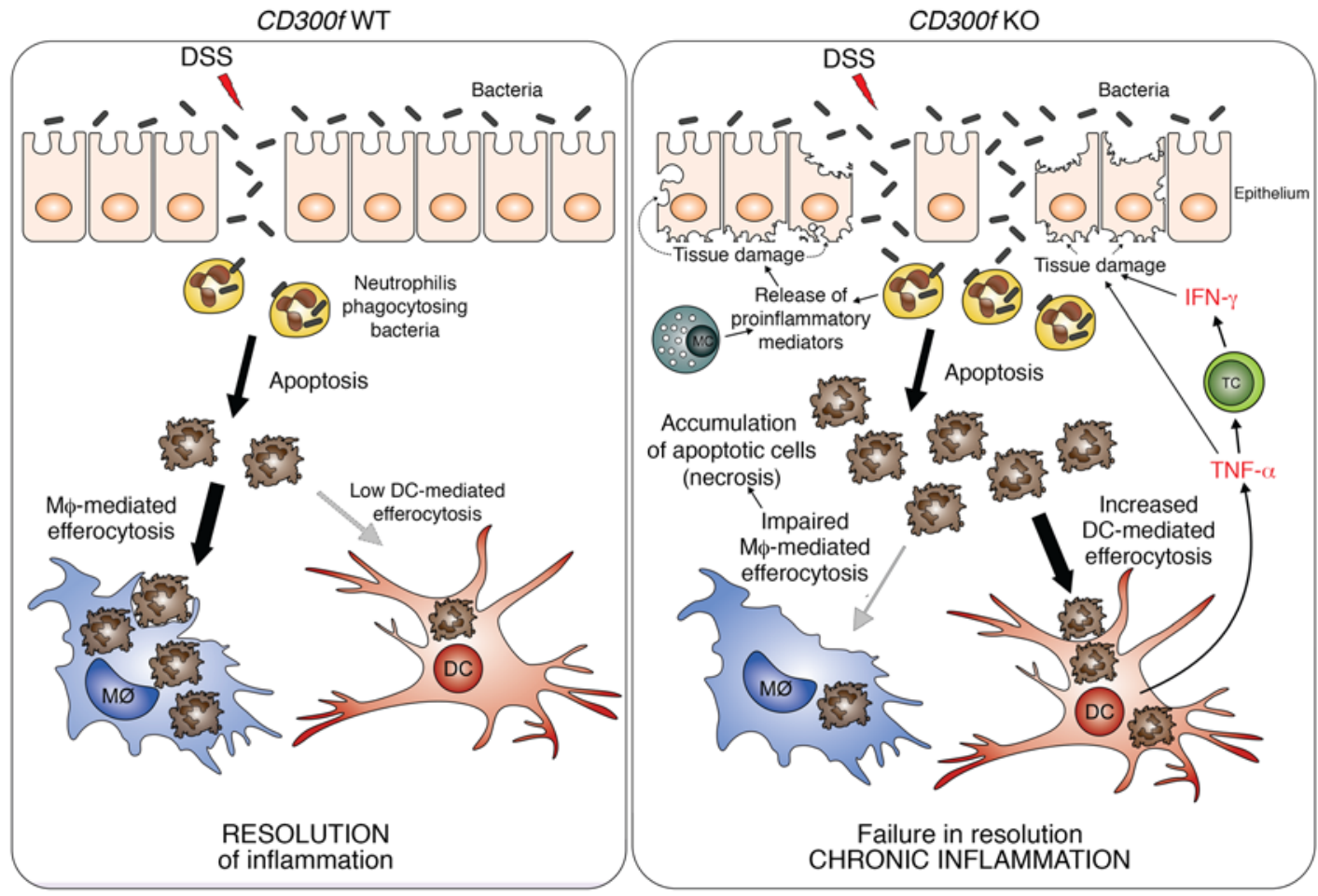

Figure 6. Aggravation of colonic inflammation in the absence of CD300f. DSS damages intestinal epithelial cells and causes intestine barrier disruption, thereby allowing bacteria to penetrate intestinal barrier. To protect the host from microbial infection, neutrophils are recruited to such inflamed sites, kill the infiltrating bacteria, and undergo apoptosis. In normal conditions (CD300f WT, left), the apoptotic neutrophils are recognized and engulfed by phagocytes (mainly macrophages, M $\phi$ ), which results in resolution of inflammation. However, under CD300f deficiency (CD300f KO, right), macrophages fail to efficiently engulf $A C s$, leading to accumulation and increased availability of $A C s$ to CD300f-deficient DCs that inherently have increased efferocytic potential. Subsequently, AC-engulfing DCs produce TNF- $\alpha$, which stimulates T cells (TC) and possibly other immune or nonimmune cells, to produce IFN- $\gamma$. Elevated levels of TNF- $\alpha$ and IFN- $\gamma$ further damage the intestinal barrier, allowing for more bacteria to enter, resulting in prolonged recruitment of neutrophils to fight the microbial intrusion, which further contributes to sustained and elevated apoptosis and accumulation of ACs. In addition, chemoattractants and proinflammatory mediators released by activated neutrophils and mast cells (MC) result in further tissue damage and sustained recruitment of immune cells to the inflamed site, thus aggravating the intestinal inflammation. Eventually, a continuous proinflammatory feedback loop is generated, leading to chronic inflammation. Therefore, CD300f is important for preventing chronic inflammation in the gut by suppressing overactive DC inflammatory responses, and ensuring proper efferocytosis.

1 for $1 \%-5 \%$ loss, 2 for $5 \%-10 \%$ loss, 3 for $10 \%-15 \%$ loss, and 4 for $>15 \%$ weight loss. Stool consistency was scored 0 for formed and hard, 1 for formed but soft, 2 for loose stool but not sticking to the anus, 3 for mild diarrhea, and 4 for gross diarrhea. For rectal bleeding, a value of 0 was assigned for none, 2 for moderate, and 4 for gross bleeding. Macroscopic assessment of colitis was graded from 0 to 5: 0 for no ulcer or inflammation, 1 for ulceration without hyperemia, 2 for ulceration and local hyperemia, 3 for ulceration and inflammation at 1 site only, 4 for more than 2 sites of ulceration and inflammation, and 5 for ulceration extending more than $2 \mathrm{~cm}$.

Histopathological analysis. Colon tissues were fixed in $10 \%$ formalin, and paraffin-embedded colon sections were stained with $\mathrm{H} \& \mathrm{E}$. Stained sections were scanned by a ScanScope XT (Aperio Technologies) and analyzed using Aperio Image Scope software (version 11). Microscopic assessment of inflammation and epithelial damage was evaluated as 0 for normal appearance with intact epithelial crypts in the mucosa, 1 for loss or disruption of the basal one-third of the crypts with mild inflammation in the mucosa, 2 for loss or disruption of the basal two-thirds of the crypts with moderate inflammation in the mucosa, 3 for loss or disruption of entire crypts with severe inflammation in the mucosa and submucosa, but retaining of the surface epithelium, and 4 for presence of mucosal ulcer with severe inflammation (infiltration of neutrophils and lymphocytes) in the mucosa, submucosa, and/or lamina propria.

Isolation of murine lamina propria immune cells from colonic tissues. Isolation of colonic lamina propria cells was performed as previously described (55). Entire colons from each group were longitudinally cut and washed to remove feces. They were then cut into $1-\mathrm{cm}$ pieces, followed by incubation in RPMI 1640 media (Lonza) containing 5 mM EDTA (Sigma-Aldrich), $0.145 \mathrm{mg} / \mathrm{ml}$ DTT (Sigma-Aldrich), and 3\% FBS (SigmaAldrich) at $37^{\circ} \mathrm{C}$ for 20 minutes on a shaking platform. After removal of EDTA by 3 washes in PBS and passing through a cell strainer $(70 \mu \mathrm{m})$, the suspension of epithelial, subepithelial, and villus cells was removed. The remaining colon pieces including lamina propria cells and muscle layer were homogenized and then incubated in digestion media containing 0.1 $\mathrm{mg} / \mathrm{ml}$ Liberase TL (Sigma-Aldrich) and 0.05\% DNase I (Sigma-Aldrich) for 25 minutes at $37^{\circ} \mathrm{C}$ on a shaking platform. After digestion, the lamina propria cells were enriched using Percoll density gradient centrifugation. The resulting cells were then used for flow cytometry analysis.

Flow cytometry. Lamina propria cells were treated with brefeldin A (eBioscience) for 3 hours, and then stained with a cocktail of antibodies against various lineage markers, including CD45 (30-F11), 
F4/80 (BM8), CD11b (M1/70), CD11c (N418), CD14 (Sa2-8), Ly6G (1A8), TCR $\beta$ (H57-597), NK1.1 (PK136), CD19 (6D5), CD4 (RM4-5), CD8a (53-6.7), FceRI (MAR-I), CD103 (2E7), B220 (RA3-6B2), and CD64 (X54-5/7.1). For intracellular staining, cells were fixed with 2\% paraformaldehyde for 12 minutes on ice, permeabilized using $0.5 \%$ saponin in PBS, and stained with antibodies specific to TNF- $\alpha$ (MP6XT22), IFN- $\gamma$ (XMG1.2), or transcription factor FoxP3 (FJK-16s) for 1 hour on ice. All of those antibodies were purchased from Biolegend. Flow cytometry data were collected with the LSR II (BD Biosciences), and the results were analyzed using FlowJo software (version 10).

Preparation of BMM $\phi$ and BMDCs. Bone marrow (BM) cells were isolated from femurs and tibias of $C D 300 f^{7 /+}$ and $C D 300 f^{/-}$mice. BMM $\phi$ differentiation was induced by culturing of BM cells in RPMI 1640 medium supplemented with 10\% FBS and 30\% L929-conditioned medium (a source of M-CSF) for 7 days with 1 addition of fresh culture medium containing 30\% L929-conditioned medium. On day 7, after removal of nonadherent cells, $\mathrm{BMM} \phi$ were detached from the plate using Accutase (Innovative Cell Technologies) and used for the further experiments. BMDC differentiation was induced by culturing of BM cells in RPMI 1640 medium supplemented with $10 \%$ FBS and $20 \mathrm{ng} / \mathrm{ml} \mathrm{GM-CSF}$ for 7 days, with 1 addition of fresh culture medium supplemented with 20 ng/ml GM-CSF. On day 7, nonadherent cells were collected, and CD11 $\mathrm{c}^{+}$ BMDCs were isolated using mouse CD11c microbeads (Miltenyi Biotec Inc.). Those purified CD11 $\mathrm{c}^{+} \mathrm{BMDC}$ s were used in the experiments.

Phagocytosis assay. Thymocytes from C57BL/6 mice were irradiated (20 Gy) and incubated in RPMI medium with 1\% BSA for 6 hours. These apoptotic thymocytes were then coincubated with either $\mathrm{BMM} \phi$ or BMDCs at a ratio 3:1 for 30 minutes, followed by incubation in a fresh medium. After 48 hours, the medium was collected and analyzed for cytokine levels. For incubation with liposomes, BMDCs were pretreated with either PBS, $10 \mu \mathrm{M}$ PS:PC liposomes, or $10 \mu \mathrm{M}$ PC:PC liposomes for 30 minutes, followed by coincubation with apoptotic thymocytes at a ratio of 1:3 for 30 minutes. Liposomes were prepared by combination of $80 \%$ synthetic 1,2-dioleoyl-sn-glycero-3phosphocholine (DOPC; Avanti Polar Lipids), and 20\% of 1-palmitoyl2-oleoyl-sn-glycero-3-phospho-L-serine (POPS; Avanti Polar Lipids) or 1-palmitoyl-2-oleoyl-sn-glycero-3-phosphocholine (POPC; Avanti Polar Lipids), as described previously (17).

Cytokine measurement. Cytokine concentrations were measured using Cytometric Bead Array mouse inflammatory kits (BD Biosciences) according to the manufacturer's instructions.

Immunofluorescence analysis. OCT-embedded colon sections $(5 \mu \mathrm{m})$ were rehydrated in PBS for 10 minutes, permeabilized with $0.1 \%$ Triton $\mathrm{X}-100$ for 5 minutes, washed in PBS twice, and blocked with 5\% BSA in PBS for 30 minutes. Phycoerythrin-conjugated anti-TNF- $\alpha$ or anti-INF- $\gamma$ antibodies, diluted 1:100 in 1\% BSA in PBS, were applied for 1 hour at room temperature. After washing twice for 5 minutes, tissue sections were treated with DAPI, mounted in ProLong Gold medium (Invitrogen), and visualized by an LSM 780 laser scanning confocal microscope (Zeiss) with a $\times 40$ Zeiss Plan-Apochromat objective. The intensities of
anti-TNF- $\alpha$ or anti-IFN- $\gamma$ staining were visualized and illustrated using ImageJ (version 1.49, NIH) and its 3D surface plot profile function.

TUNEL assay. ACs were detected by the TUNEL assay with the TUNEL Apoptosis Detection kit (GenScript) according to the manufacturer's instructions.

Gut microbiome analysis. Bacterial genomic DNA was extracted from snap-frozen mouse fecal pellets using the QIAamp DNA Stool Mini Kit (QIAGEN) according to the manufacturer's instructions. The V3 and V4 hypervariable region of the 16S rRNA gene was amplified using specific primers (515F: 5'-GTGCCAGCAGCCGCGGTAA-3'; and 806R: 5'-GGACTACCAGGGTATCTAAT-3'). 16S sequencing was carried out by paired-end sequencing of the V4 region on an Illumina MiSeq. Raw reads were processed and analyzed using the mothur MiSeq pipeline as implemented in Nephele (https://nephele.niaid.nih.gov) with default parameters and a minimum quality score of 29. Unweighted UniFrac

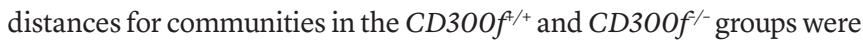
not found to be statistically significant, based on analysis of similarity (ANOSIM) test. The sequences reported in this paper have been deposited in the GenBank database (BioProject ID: PRJNA319680).

Statistics. Statistical significance was determined by the 2-tailed unpaired Student's $t$ test (GraphPad Software, version 6.0). Data are presented as mean \pm SEM; $P<0.05$ was considered to be statistically significant.

Study approval. All animal studies were conducted in accordance with the experimental procedure (LIG-5E) approved by the NIAID Animal Care and Use Committee.

\section{Author contributions}

HNL and KK designed the experiments. HNL, LT, and NB performed the experiments. JD, MQ, and YB performed the microbiome analysis. Results were analyzed by HNL, JEC, and KK. KK, HNL, and JEC wrote the paper.

\section{Acknowledgments}

We thank Aleksandra Gil-Krzewska, Herbert Morse III, Oliver Voss, and Hongsheng Wang for critically reading the manuscript. We thank Mirna Pena for handling the animal colony. We thank Joseph Brzostowski for the technical help with the microscopy. We acknowledge the NIAID Microbiome Program Microbiome Sequencing Platform for help and assistance in the microbiome analysis. This work was supported by the intramural research program of the National Institute of Allergy and Infectious Diseases, and a Korean Biomedical Scientist Fellowship Program grant (awarded to HNL) from the Korea Research Institute of Bioscience and Biotechnology.

Address correspondence to: Konrad Krzewski or John E. Coligan, Receptor Cell Biology Section, Laboratory of Immunogenetics, NIAID, NIH, 12441 Parklawn Drive, Rockville, Maryland 20852, USA. Phone: 301.761.5030; E-mail: krzewskikj@niaid.nih.gov (K. Krzewski); jcoligan@niaid.nih.gov (J.E. Coligan).
1. Cader MZ, Kaser A. Recent advances in inflammatory bowel disease: mucosal immune cells in intestinal inflammation. Gut. 2013;62(11):1653-1664.

2. Kaser A, Zeissig S, Blumberg RS. Inflammatory bowel disease. Annu Rev Immunol. 2010;28:573-621.
3. Edelblum KL, Yan F, Yamaoka T, Polk DB. Regulation of apoptosis during homeostasis and disease in the intestinal epithelium. Inflamm Bowel Dis. 2006;12(5):413-424.

4. Hagiwara C, Tanaka M, Kudo H. Increase in colorectal epithelial apoptotic cells in patients with ulcerative colitis ultimately requiring surgery. J Gastroenterol Hepatol. 2002;17(7):758-764.

5. Qiu W, et al. PUMA-mediated intestinal epithelial apoptosis contributes to ulcerative colitis in humans and mice. J Clin Invest. 2011;121(5):1722-1732. 
6. Souza HS, et al. Apoptosis in the intestinal mucosa of patients with inflammatory bowel disease: evidence of altered expression of FasL and perforin cytotoxic pathways. Int J Colorectal Dis. 2005;20(3):277-286

7. Nenci A, et al. Epithelial NEMO links innate immunity to chronic intestinal inflammation. Nature. 2007;446(7135):557-561.

8. Kaser A, et al. XBP1 links ER stress to intestinal inflammation and confers genetic risk for human inflammatory bowel disease. Cell. 2008;134(5):743-756.

9. Steinbrecher KA, Harmel-Laws E, Sitcheran R, Baldwin AS. Loss of epithelial RelA results in deregulated intestinal proliferative/apoptotic homeostasis and susceptibility to inflammation. JImmunol. 2008;180(4):2588-2599.

10. Welz PS, et al. FADD prevents RIP3-mediated epithelial cell necrosis and chronic intestinal inflammation. Nature. 2011;477(7364):330-334.

11. Hart AL, et al. Characteristics of intestinal dendritic cells in inflammatory bowel diseases. Gastroenterology. 2005;129(1):50-65.

12. te Velde AA, et al. Increased expression of $\mathrm{DC}^{-} \mathrm{SIGN}^{+} \mathrm{IL}-12^{+} \mathrm{IL}-18^{+}$and CD $83^{+} \mathrm{IL}-12-\mathrm{IL}-18$ dendritic cell populations in the colonic mucosa of patients with Crohn's disease. Eur J Immunol. 2003;33(1):143-151.

13. Baumgart DC, et al. Exaggerated inflammatory response of primary human myeloid dendritic cells to lipopolysaccharide in patients with inflammatory bowel disease. Clin Exp Immunol. 2009;157(3):423-436.

14. Ravichandran KS. Find-me and eat-me signals in apoptotic cell clearance: progress and conundrums. JExp Med. 2010;207(9):1807-1817.

15. Ravichandran KS. Beginnings of a good apoptotic meal: the find-me and eat-me signaling pathways. Immunity. 2011;35(4):445-455.

16. Choi SC, et al. Cutting edge: mouse CD300f (CMRF-35-like molecule-1) recognizes outer membrane-exposed phosphatidylserine and can promote phagocytosis. J Immunol. 2011;187(7):3483-3487.

17. Tian L, et al. p85 $\alpha$ recruitment by the CD300f phosphatidylserine receptor mediates apoptotic cell clearance required for autoimmunity suppression. Nat Commun. 2014;5:3146.

18. Tian L, et al. Enhanced efferocytosis by dendritic cells underlies memory T-cell expansion and susceptibility to autoimmune disease in CD300f-deficient mice. Cell Death Differ. 2016;23(6):1086-1096.

19. Perše M, Cerar A. Dextran sodium sulphate colitis mouse model: traps and tricks. J Biomed Biotechnol. 2012;2012:718617.

20. Matsukawa T, et al. Ceramide-CD300f binding suppresses experimental colitis by inhibiting ATP-mediated mast cell activation. Gut. 2016;65(5):777-787.

21. Fries $\mathrm{W}$, et al. Infliximab and etanercept are equally effective in reducing enterocyte APOPTOSIS in experimental colitis. Int J Med Sci. 2008;5(4):169-180.

22. Deveci F, Muz MH, Ilhan N, Kirkil G, Turgut T, Akpolat N. Evaluation of the anti-inflammatory effect of infliximab in a mouse model of acute asthma. Respirology. 2008;13(4):488-497.

23. Wang F, Graham WV, Wang Y, Witkowski ED, Schwarz BT, Turner JR. Interferon- $\gamma$ and tumor necrosis factor- $\alpha$ synergize to induce intestinal epithelial barrier dysfunction by up-regulating myosin light chain kinase expression. Am J Pathol. 2005;166(2):409-419.

24. Hochreiter-Hufford A, Ravichandran KS. Clearing the dead: apoptotic cell sensing, recognition, engulfment, and digestion. Cold Spring Harb Perspect Biol. 2013;5(1):a008748.

25. Fournier BM, Parkos CA. The role of neutrophils during intestinal inflammation. Mucosal Immunol. 2012;5(4):354-366.

26. Chung EY, et al. Interleukin-10 expression in macrophages during phagocytosis of apoptotic cells is mediated by homeodomain proteins $\mathrm{Pbx} 1$ and Prep-1. Immunity. 2007;27(6):952-964.

27. Marlow GJ, van Gent D, Ferguson LR. Why interleukin-10 supplementation does not work in Crohn's disease patients. World J Gastroenterol. 2013;19(25):3931-3941.

28. Medzhitov R. Origin and physiological roles of inflammation. Nature. 2008;454(7203):428-435

29. Lee HN, Na HK, Surh YJ. Resolution of inflammation as a novel chemopreventive strategy. Semin Immunopathol. 2013;35(2):151-161.

30. Berndt BE, Zhang M, Chen GH, Huffnagle GB, Kao JY. The role of dendritic cells in the development of acute dextran sulfate sodium colitis. J Immunol. 2007;179(9):6255-6262.

31. Tlaskalová-Hogenová $\mathrm{H}$, et al. Involvement of innate immunity in the development of inflammatory and autoimmune diseases. Ann N Y Acad Sci. 2005;1051:787-798.

32. Szondy Z, Garabuczi E, Joós G, Tsay GJ, Sarang Z. Impaired clearance of apoptotic cells in chronic inflammatory diseases: therapeutic implications. Front Immunol. 2014;5:354.

33. Mahida YR. The key role of macrophages in the immunopathogenesis of inflammatory bowel disease. Inflamm Bowel Dis. 2000;6(1):21-33.

34. Moshkovits I, et al. A key requirement for $\mathrm{CD} 300 \mathrm{f}$ in innate immune responses of eosinophils in colitis. Mucosal Immunol. 2017;10(1):172-183.

35. Chung DH, Humphrey MB, Nakamura MC, Ginzinger DG, Seaman WE, Daws MR. CMRF35-like molecule-1, a novel mouse myeloid receptor, can inhibit osteoclast formation. J Immunol. 2003;171(12):6541-6548.

36. Clark GJ, Ju X, Tate C, Hart DN. The CD300 family of molecules are evolutionarily significant regulators of leukocyte functions. Trends Immunol. 2009;30(5):209-217.

37. Moshkovits I, et al. CD300f associates with IL-4 receptor $\alpha$ and amplifies IL-4-induced immune cell responses. Proc Natl Acad Sci U S A. 2015;112(28):8708-8713.

38. Shik D, Moshkovits I, Karo-Atar D, Reichman H, Munitz A. Interleukin-33 requires CMRF35-like molecule-1 expression for induction of myeloid cell activation. Allergy. 2014;69(6):719-729.

39. Moshkovits I, et al. CD300f associates with IL-4 receptor $\alpha$ and amplifies IL-4-induced immune cell responses. Proc Natl Acad Sci U S A. 2015;112(28):8708-8713.
40. Bates J, Diehl L. Dendritic cells in IBD pathogenesis: an area of therapeutic opportunity? J Pathol. 2014;232(2):112-120.

41. Qualls JE, Tuna H, Kaplan AM, Cohen DA. Suppression of experimental colitis in mice

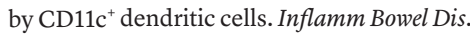
2009;15(2):236-247.

42. Chogle A, Bu HF, Wang X, Brown JB, Chou PM, Tan XD. Milk fat globule-EGF factor 8 is a critical protein for healing of dextran sodium sulfateinduced acute colitis in mice. Mol Med. 2011;17(5-6):502-507.

43. Aziz MM, et al. MFG-E8 attenuates intestinal inflammation in murine experimental colitis by modulating osteopontin-dependent $\alpha v \beta 3$ integrin signaling. J Immunol. 2009;182(11):7222-7232.

44. Bosurgi L, et al. Paradoxical role of the proto-oncogene Axl and Mer receptor tyrosine kinases in colon cancer. Proc Natl Acad Sci U S A. 2013;110(32):13091-13096.

45. Izawa K, et al. Functional analysis of activating receptor LMIR 4 as a counterpart of inhibitory receptor LMIR3. J Biol Chem. 2007;282(25):17997-18008.

46. Izawa $\mathrm{K}$, et al. An activating and inhibitory signal from an inhibitory receptor LMIR3/CLM-1: LMIR3 augments lipopolysaccharide response through association with FcR $\gamma$ in mast cells. J Immunol. 2009;183(2):925-936.

47. Moshkovits I, et al. CMRF35-like molecule 1 (CLM-1) regulates eosinophil homeostasis by suppressing cellular chemotaxis. Mucosal Immunol. 2014;7(2):292-303.

48. Chung DH, Humphrey MB, Nakamura MC, Ginzinger DG, Seaman WE, Daws MR. CMRF35-like molecule-1, a novel mouse myeloid receptor, can inhibit osteoclast formation. JImmunol. 2003;171(12):6541-6548.

49. Michlewska S, Dransfield I, Megson IL, Rossi AG. Macrophage phagocytosis of apoptotic neutrophils is critically regulated by the opposing actions of pro-inflammatory and anti-inflammatory agents: key role for TNF- $\alpha$. FASEB J . 2009;23(3):844-854.

50. Neurath MF. Cytokines in inflammatory bowel disease. Nat Rev Immunol. 2014;14(5):329-342.

51. Galli SJ, Borregaard N, Wynn TA. Phenotypic and functional plasticity of cells of innate immunity: macrophages, mast cells and neutrophils. Nat Immunol. 2011;12(11):1035-1044.

52. Ramsay DB, Stephen S, Borum M, Voltaggio L, Doman DB. Mast cells in gastrointestinal disease. Gastroenterol Hepatol (N Y). 2010;6(12):772-777.

53. Bian Z, Guo Y, Ha B, Zen K, Liu Y. Regulation of the inflammatory response: enhancing neutrophil infiltration under chronic inflammatory conditions. JImmunol. 2012;188(2):844-853.

54. Lopetuso LR, et al. Locally injected Infliximab ameliorates murine DSS colitis: differences in serum and intestinal levels of drug between healthy and colitic mice. Dig Liver Dis. 2013;45(12):1017-1021.

55. Yagi $R$, et al. The transcription factor GATA3 is critical for the development of all IL-7R $\alpha$ expressing innate lymphoid cells. Immunity. 2014;40(3):378-388. 